\title{
Hyperspectral Super-Resolution with Coupled Tucker Approximation: Recoverability and SVD-based algorithms
}

\author{
Clémence Prévost, Student Member, IEEE, Konstantin Usevich*, Member, IEEE, \\ Pierre Comon, Fellow, IEEE, and David Brie Member, IEEE,
}

\begin{abstract}
We propose a novel approach for hyperspectral super-resolution, that is based on low-rank tensor approximation for a coupled low-rank multilinear (Tucker) model. We show that the correct recovery holds for a wide range of multilinear ranks. For coupled tensor approximation, we propose two SVD-based algorithms that are simple and fast, but with a performance comparable to the state-of-the-art methods. The approach is applicable to the case of unknown spatial degradation and to the pansharpening problem.
\end{abstract}

Index Terms-hyperspectral super-resolution, low-rank tensor approximation, data fusion, recovery, identifiability

\section{INTRODUCTION}

The problem of hyperspectral super-resolution (HSR) [1] has recenlty attracted much interest from the signal processing community. It consists in fusing a multispectral image (MSI), which has a good spatial resolution but few spectral bands, and a hyperspectral image (HSI), whose spatial resolution is lower than that of MSI. The aim is to recover a superresolution image (SRI), which possesses both good spatial and spectral resolutions. This problem is closely related to hyperspectral pansharpening [2], [3], where the HSI is fused with a panchromatic image (i.e. an "MSI" with one spectral band).

Many methods were developed for the HSR problem, including coupled nonnegative matrix factorization [4] (CNMF), methods based on solving Sylvester equations [5], Bayesian approaches (HySure [6]), FUMI [7], to name a few. Motivated by the widely used linear mixing model, most of these methods are based on a coupled low-rank factorization of the matricized hyperspectral and multispectral images. In [8], a matrix factorization approach under sparsity conditions was proposed, together with a proof of correct recovery of the estimated SRI in the noiseless case.

Recently, a promising tensor-based method was proposed that makes use of the inherent 3D nature of HSI [9]. Assuming that the super-resolution image itself admits a low-rank

This work was partially supported by the ANR (Agence Nationale de Recherche) under grants OPTIFIN (ANR-15-CE10-0007) and LeaFleT (ANR19-CE23-0021).

C. Prévost, K. Usevich and D. Brie are with Centre de Recherche en Automatique de Nancy (CRAN), Université de Lorraine, CNRS, Boulevard des Aiguillettes, BP 70239, F-54506 Vandoeuvre-lès-Nancy, France. P. Comon is with CNRS, GIPSA-Lab, Univ. Grenoble Alpes, F-38000 Grenoble, France.

Email: clemence.prevost@univ-lorraine.fr, konstantin.usevich@univlorraine.fr, pierre.comon@gipsa-lab.fr, david.brie@univ-lorraine.fr. Fax: +33 383684437. Tel.: +33 372745313 (K. Usevich).

*Corresponding author. canonical polyadic (CP) decomposition (CPD), the HSR is reformulated as a coupled $\mathrm{CP}$ approximation. An alternating least squares (ALS) algorithm called Super-resolution TEnsor REconstruction (STEREO) is proposed, achieving reconstruction performance that is competitive with the state of the art. A proof of the correct recovery of the SRI by the approach of [9] is given provided the CPD of the MSI is unique. This approach was also successfully used for a super-resolution problem in medical imaging [10].

In some cases, the spatial degradation operator is unknown, therefore blind algorithms are needed. A blind version of STEREO was proposed in [9] that also uses an ALS algorithm for a coupled CP model. In [11], a simple Super-resolution CUBe Algorithm (SCUBA) based on a single CPD of the MSI tensor and a truncated SVD of the unfolding of the HSI is introduced. A key idea proposed in [11] is to use local approximations by splitting the data cubes into separate blocks. This algorithm outperforms blind STEREO and other state-ofthe-art algorithms. It also does not require separability of the spatial degradation operator.

In this paper, we propose to use another type of lowrank tensor factorization: multilinear (also known as Tucker) factorization. By assuming that the super-resolution image has approximately low multilinear rank, we reformulate the HSR problem as a coupled Tucker approximation. First, we propose two closed-form SVD-based algorithms: the first, named Super-resolution based on COupled Tucker Tensor approximation (SCOTT), is inspired by the higher-order SVD [12] and the second (blind) is inspired by [11]. Second, we prove that, although the Tucker decomposition is not identifiable, the SRI can be uniquely recovered for a wide range of multilinear ranks. While the proposed exact recovery conditions are in general more restrictive than those of [9], they can be specialized in situations for which nothing can be concluded from [9]. Our experiments on a number of simulated and semi-real examples, show that the proposed algorithms have a performance approaching those of [9] and [11], but the computational cost is much lower. Also, the proposed approach is applicable to hyperspectral pansharpening [13] (unlike [9], which requires the MSI to have at least two spectral bands). Finally, the algorithms can accurately reconstruct spectral signatures, which is of prime importance for further processing of the HSR image.

A short version of this work [14] appears in ICASSP 2019, presenting the SCOTT algorithm and part of the simulations. 
The current paper additionally includes new blind algorithms, detailed analysis of the model and the algorithms, proof of the theorem for recoverability, new simulations for synthetic and semi-real data, examples on recovery of spectral signatures.

This paper is organized as follows. In Section III we introduce our notation, define basic tensor decomposition operations and recall the HSR problem. In Section III, we recall the CP-based model and the STEREO algorithm proposed in [9]. Section IV] contains our proposed coupled Tucker model and SVD-based algorithms (SCOTT and B-SCOTT) for tensor approximation. In Section V/we prove our main recoverability result for the coupled Tucker model. Section VI contains the numerical experiments.

\section{BACKGROUND AND NOTATION}

\section{A. Basic notation}

In this paper we mainly follow [15] in what concerns the tensor notation (see also [16]). The following fonts are used: lowercase $(a)$ or uppercase $(A)$ plain font for scalars, boldface lowercase $(\boldsymbol{a})$ for vectors, uppercase boldface $(\boldsymbol{A})$ for matrices, and calligraphic $(\mathcal{A})$ for $N$-D arrays (tensors). Vectors are, by convention, one-column matrices. The elements of vectors/matrices/tensors are accessed as $a_{i}, A_{i, j}$ and $\mathcal{A}_{i_{1}, \ldots, i_{N}}$ respectively. $\mathbb{R}$ stands for the real line.

For a matrix $\boldsymbol{A}$, we denote its transpose and Moore-Penrose pseudoinverse as $\boldsymbol{A}^{\top}$ and $\boldsymbol{A}^{\dagger}$ respectively. The notation $\boldsymbol{I}_{M}$ is used for the $M \times M$ identity matrix and $\mathbf{0}_{L \times K}$ for the $L \times K$ matrix of zeroes. We use the symbol $\otimes$ for the Kronecker product of matrices (in order to distinguish it from the tensor product $\otimes)$, and $\odot$ for the Khatri-Rao product.

For a matrix $\boldsymbol{X} \in \mathbb{R}^{m \times n}$, we denote by $\sigma_{\max }(\boldsymbol{X})$ and $\sigma_{\min }(\boldsymbol{X})$ the largest and the smallest of the $\min (m, n)$ singular values of $\boldsymbol{X}$. We also denote by $\operatorname{tSVD}_{R}(\boldsymbol{X}) \in \mathbb{R}^{n \times R}$ a matrix containing $R$ leading right singular vectors of $\boldsymbol{X}$.

We use vec $\{\cdot\}$ for the standard column-major vectorization of a tensor or a matrix. Operator $\bullet_{p}$ denotes contraction on the $p$ th index of a tensor; when contracted with a matrix, summation is always performed on the second index of the matrix, e.g., $\left[\mathcal{A} \bullet_{1} \boldsymbol{M}\right]_{i j k}=\sum_{\ell} \mathcal{A}_{\ell j k} M_{i \ell}$. For a tensor $\mathcal{Y} \in \mathbb{R}^{I \times J \times K}$, its first unfolding is denoted by $\boldsymbol{Y}^{(1)} \in \mathbb{R}^{J K \times I}$.

\section{B. Tensor decompositions}

For a tensor $\mathcal{G} \in \mathbb{R}^{R_{1} \times R_{2} \times R_{3}}$ and matrices $\boldsymbol{U} \in \mathbb{R}^{I \times R_{1}}$, $\boldsymbol{V} \in \mathbb{R}^{J \times R_{2}}$ and $\boldsymbol{W} \in \mathbb{R}^{K \times R_{3}}$, the following shorthand notation is used for the multilinear product:

$$
\llbracket \mathcal{G} ; \boldsymbol{U}, \boldsymbol{V}, \boldsymbol{W} \rrbracket=\mathcal{G} \underset{1}{\bullet} \boldsymbol{U} \underset{2}{\bullet} \boldsymbol{V} \bullet \boldsymbol{W} .
$$

which means that the $(i, j, k)$ th entry of the above array is

$$
\sum_{p q r} G_{p q r} U_{i p} V_{j q} W_{k r}
$$

If $\mathcal{Y}=\llbracket \mathcal{G} ; \boldsymbol{U}, \boldsymbol{V}, \boldsymbol{W} \rrbracket$, the following identities hold for its vectorization and unfoldings, respectively:

$$
\begin{aligned}
\operatorname{vec}\{\mathcal{Y}\} & =(\boldsymbol{W} \otimes \boldsymbol{V} \otimes \boldsymbol{U}) \operatorname{vec}\{\mathcal{G}\}, \\
\boldsymbol{Y}^{(1)} & =(\boldsymbol{W} \otimes \boldsymbol{V}) \boldsymbol{G}^{(1)} \boldsymbol{U}^{\top} .
\end{aligned}
$$

If, in addition,

$$
R_{1}=\operatorname{rank}\left\{\boldsymbol{Y}^{(1)}\right\}, R_{2}=\operatorname{rank}\left\{\boldsymbol{Y}^{(2)}\right\}, R_{3}=\operatorname{rank}\left\{\boldsymbol{Y}^{(3)}\right\},
$$

then the multilinear product is called Tucker decomposition of $\mathcal{Y}$ and $\left(R_{1}, R_{2}, R_{3}\right)$ are called the multilinear ranks.

For matrices $\boldsymbol{A} \in \mathbb{R}^{I \times F}, \boldsymbol{B} \in \mathbb{R}^{J \times F}, \boldsymbol{C} \in \mathbb{R}^{K \times F}$, we will use a shorthand notation for a polyadic decomposition (sometimes also called rank-decomposition)

$$
\llbracket \boldsymbol{A}, \boldsymbol{B}, \boldsymbol{C} \rrbracket=\llbracket \mathcal{I}_{F} ; \boldsymbol{A}, \boldsymbol{B}, \boldsymbol{C} \rrbracket,
$$

where $\mathcal{I}_{F} \in \mathbb{R}^{F \times F \times F}$ is a diagonal tensor of ones. In other words, if $\mathcal{Y}=\llbracket \boldsymbol{A}, \boldsymbol{B}, \boldsymbol{C} \rrbracket$, then

$$
\mathcal{Y}_{i j k}=\sum_{r} A_{i r} B_{j r} C_{k r} ;
$$

moreover, the first unfolding can be expressed as

$$
\boldsymbol{Y}^{(1)}=(\boldsymbol{C} \odot \boldsymbol{B}) \boldsymbol{A}^{\top}
$$

Finally, if $F$ is minimal, $\mathcal{Y}=\llbracket A, B, C \rrbracket$ is called canonical polyadic (CP) decomposition and $F$ is called the tensor rank.

\section{Hyperspectral super-resolution and degradation model}

We consider a multispectral image (MSI) cube $\mathcal{Y}_{M} \in$ $\mathbb{R}^{I \times J \times K_{M}}$ and a hyperspectral image (HSI) cube $\mathcal{Y}_{H} \in$ $\mathbb{R}^{I_{H} \times J_{H} \times K}$ acquired from existing sensors (for instance, LANDSAT or QuickBird). The spectral resolution of the MSI is lower than that of the HSI $\left(K_{M} \ll K\right)$, while its spatial resolution is higher $\left(I>I_{H}, J>J_{H}\right)$. The acquired MSI and HSI usually represent the same target, and $\mathcal{Y}_{M}$ and $\mathcal{Y}_{H}$ are viewed as two degraded versions of a single super-resolution image (SRI) data cube $\mathcal{Y} \in \mathbb{R}^{I \times J \times K}$. The hyperspectral data fusion problem [1] consists in recovering SRI $\mathcal{Y}$ from $\mathcal{Y}_{M}$ and $\mathcal{Y}_{H}$.

In this paper, as in [9], we adopt the following degradation model, that can be compactly written as contraction of SRI:

$$
\left\{\begin{array}{l}
\mathcal{Y}_{M}=\mathcal{Y} \bullet_{3} \boldsymbol{P}_{M}+\mathcal{E}_{M}, \\
\mathcal{Y}_{H}=\mathcal{Y} \bullet_{1} \boldsymbol{P}_{1} \bullet_{2} \boldsymbol{P}_{2}+\mathcal{E}_{H},
\end{array}\right.
$$

where $\mathcal{E}_{M}, \mathcal{E}_{H}$ denote the noise terms, $\boldsymbol{P}_{M} \in \mathbb{R}^{K_{M} \times K}$ is the spectral degradation matrix (for example, a selection-averaging matrix), and $\boldsymbol{P}_{1} \in \mathbb{R}^{I_{H} \times I}, \boldsymbol{P}_{2} \in \mathbb{R}^{J_{H} \times J}$ are the spatial degradation matrices, i.e. we assume (for simplicity) that the spatial degradation is separable. For example, the commonly accepted Wald's protocol [17] uses separable Gaussian blurring and downsampling in both spatial dimensions.

\section{CP-BASED DATA FUSION}

In [9] it was proposed to model the SRI data cube as a tensor with low tensor rank, i.e. $\mathcal{Y}=\llbracket \boldsymbol{A}, \boldsymbol{B}, \boldsymbol{C} \rrbracket$, where $\boldsymbol{A} \in \mathbb{R}^{I \times F}$, $\boldsymbol{B} \in \mathbb{R}^{J \times F}$ and $\boldsymbol{C} \in \mathbb{R}^{K \times F}$ are the factor matrices of the $\mathrm{CPD}$ and $F$ is the tensor rank. 


\section{A. The case of known spatial degradation (STEREO)}

In this subsection, we consider only the case when the degradation matrices $\boldsymbol{P}_{1}, \boldsymbol{P}_{2}, \boldsymbol{P}_{M}$ are known; the case of unknown degradation matrices is postponed to Section [III-B In this case, the HSR problem can be formulated as

$$
\underset{\widehat{A}, \widehat{B}, \widehat{C}}{\operatorname{minimize}} f_{C P}(\widehat{\boldsymbol{A}}, \widehat{\boldsymbol{B}}, \widehat{\boldsymbol{C}}),
$$

where $f_{C P}(\widehat{\boldsymbol{A}}, \widehat{\boldsymbol{B}}, \widehat{\boldsymbol{C}})=$

$$
\left\|\mathcal{Y}_{H}-\llbracket \boldsymbol{P}_{1} \widehat{\boldsymbol{A}}, \boldsymbol{P}_{2} \widehat{\boldsymbol{B}}, \widehat{\boldsymbol{C}} \rrbracket\right\|_{F}^{2}+\lambda\left\|\mathcal{Y}_{M}-\llbracket \widehat{\boldsymbol{A}}, \widehat{\boldsymbol{B}}, \boldsymbol{P}_{M} \widehat{\boldsymbol{C}} \rrbracket\right\|_{F}^{2},
$$

which is a coupled CP approximation problem. As in [9] we set $\lambda=1$ so that both degradated images have the same weight in the cost function. Thus, we consider that the HSI and MSI share the same level of additive noise. For the case when there is no noise $\left(\mathcal{E}_{H}, \mathcal{E}_{M}=\mathbf{0}\right)$, the coupled $\mathrm{CP}$ model is (generically) identifiable if

$$
F \leq \min \left\{2^{\left\lfloor\log _{2}\left(K_{M} J\right)\right\rfloor-2}, I_{H} J_{H}\right\},
$$

see [9] for a proof and details of this condition.

To solve (3), an alternating optimization algorithm is proposed in [9], called STEREO, as it is described in Algorithm 1 .

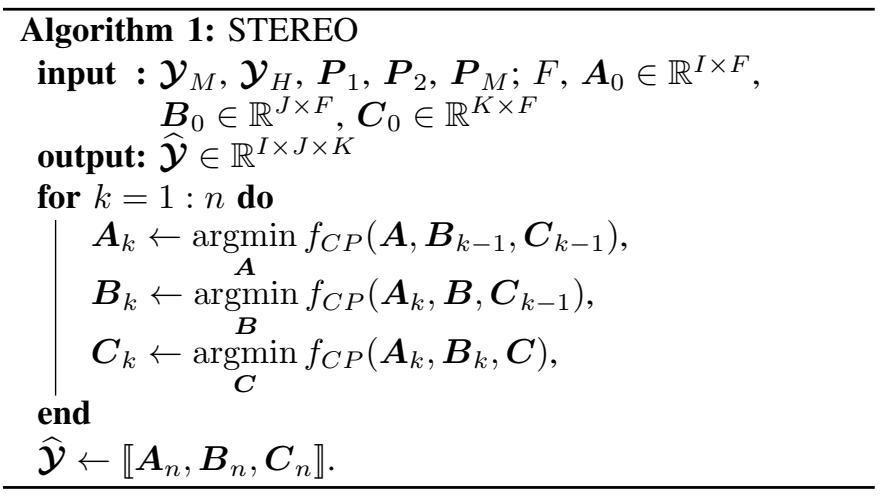

The updates of the factor matrices in Algorithm 1 can be computed by using efficient solvers for the (generalized) Sylvester equation [18], [19]. For example, the total cost of one iteration (updating $\boldsymbol{A}, \boldsymbol{B}, \boldsymbol{C})$ in Algorithm 1 becomes

- $O\left(I J K_{M} F+I_{H} J_{H} K F\right)$ flops for computing the right hand sides in the least-squares subproblems.

- $O\left(I^{3}+J^{3}+K^{3}+F^{3}\right)$ flops for solving Sylvester equations;

For more details on solving Sylvester equations, $\operatorname{se}^{1}[9$, Appendix E] and Appendix A. The initial values in Algorithm 1 are chosen as in Algorithm 2 .

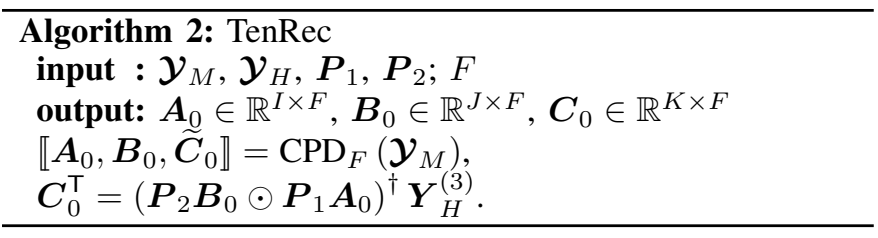

\footnotetext{
${ }^{1}$ Note that in [9 Appendix E] the cost of solving the Sylvester equation is stated as $O\left(I^{3}\right)$ and not $O\left(I^{3}+F^{3}\right)$ as in [18].
}

where $\mathrm{CPD}_{F}\left(\mathcal{Y}_{M}\right)$ stands for a rank- $F \mathrm{CP}$ approximation ${ }^{2}$ of $\mathcal{Y}_{M}$, and $C_{0}$ is obtained by solving a least-squares problem. Algorithm 2 can be used as an algebraic method for solving the HSR problem.

\section{B. The case of unknown spatial degradation}

In this subsection, we recall the CP-based methods for the HSR problem in the case when the spatial degradation matrices $\boldsymbol{P}_{1}, \boldsymbol{P}_{2}$ are unknown, proposed in [9] and [11]. The first solution, called Blind-STEREO was to consider the following coupled CP approximation problem:

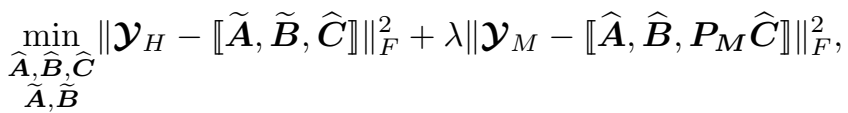

where the estimated SRI is computed as $\widehat{\mathcal{Y}}=\llbracket \widehat{A}, \widehat{B}, \widehat{C} \rrbracket$ and the matrices $\widetilde{\boldsymbol{A}}, \widetilde{\boldsymbol{B}}$ represent degraded versions of $\widehat{A}$, $\widehat{B}$ by unknown spatial degradation matrices, respectively. The conditions for correct recovery were established in [9].

In [11], an alternative approach was proposed, that uses standard CP approximation of $\mathcal{Y}_{M}$ together with an SVD of $\boldsymbol{Y}_{H}^{(3)}$, and a least squares problem. This approach, which does not necessary need separability of the spatial degradation operation, is summarized in Algorithm 3

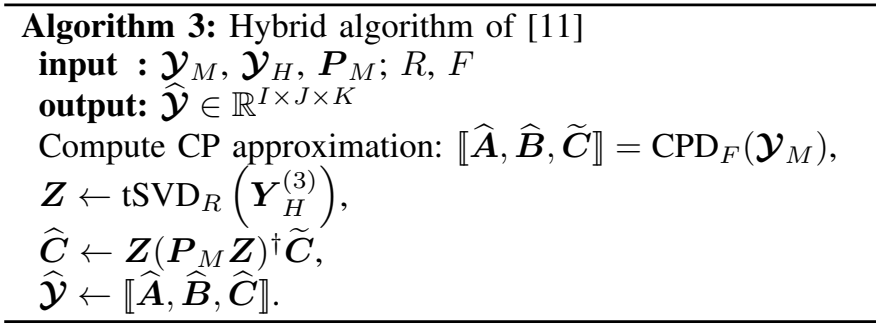

As noted in [11], $\mathcal{Y}=\llbracket A, B, C \rrbracket$ can be uniquely recovered only if $\operatorname{rank}\{\boldsymbol{C}\}=R$ does not exceed the number $K_{M}$ of spectral bands in the MSI. To overcome this limitation, in [11] it was proposed to apply Algorithm 3 to corresponding non-overlapping subblocks of the MSI and HSI (based on the hypothesis that only a small number of materials are active in a smaller block). This is summarized in Algorithm 4 called SCUBA in [11]. It was shown in [11] that such an algorithm outperforms Blind-STEREO, and other state-of-theart algorithms for blind HSR.

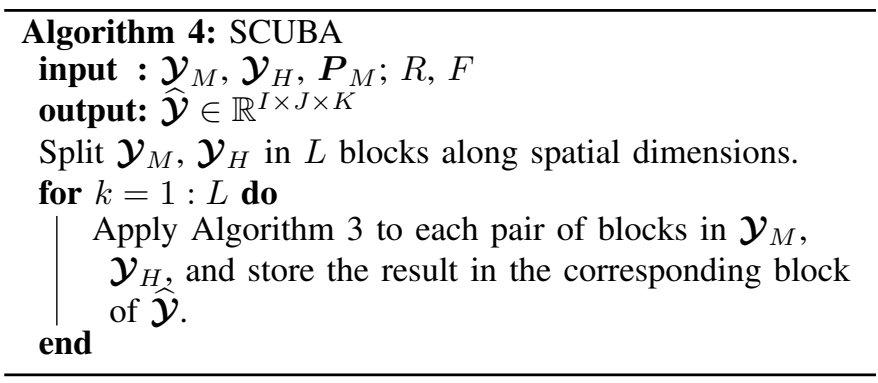

${ }^{2} \mathrm{~A}$ low tensor rank approximation does not always exist in general, but is guaranteed to exist if all terms are imposed to be entry-wise nonnegative [20]. 


\section{TUCKER-BASED DATA FUSION}

\section{A. Model and approximation problem}

In this paper, we propose a Tucker-based coupled mode ${ }^{3}$ as an alternative to STEREO. Let $\boldsymbol{R}=\left(R_{1}, R_{2}, R_{3}\right)$ be the multilinear ranks of the SRI $\mathcal{Y}$, and let $\mathcal{Y}=\llbracket \mathcal{G} ; \boldsymbol{U}, \boldsymbol{V}, \boldsymbol{W} \rrbracket$ be its Tucker decomposition, where $\boldsymbol{U} \in \mathbb{R}^{I \times R_{1}}, \boldsymbol{V} \in \mathbb{R}^{J \times R_{2}}$ and $\boldsymbol{W} \in \mathbb{R}^{K \times R_{3}}$ are the factor matrices and $\mathcal{G} \in \mathbb{R}^{R_{1} \times R_{2} \times R_{3}}$ is the core tensor.

With these notations, Equation (2) becomes

$$
\left\{\begin{array}{l}
\mathcal{Y}_{M}=\llbracket \mathcal{G} ; \boldsymbol{U}, \boldsymbol{V}, \boldsymbol{P}_{\boldsymbol{M}} \boldsymbol{W} \rrbracket+\mathcal{E}_{M}, \\
\mathcal{Y}_{H}=\llbracket \mathcal{G} ; \boldsymbol{P}_{\mathbf{1}} \boldsymbol{U}, \boldsymbol{P}_{\mathbf{2}} \boldsymbol{V}, \boldsymbol{W} \rrbracket+\mathcal{E}_{H},
\end{array}\right.
$$

thus the HSR task can be performed by estimating ${ }^{4}$ the factor matrices $\boldsymbol{U}, \boldsymbol{V}, \boldsymbol{W}$ and the core tensor $\mathcal{G}$ in the Tucker decomposition of the SRI.

As in Section III. one of the possible ways is to reformulate the HSR problem as an optimization problem:

$$
\begin{aligned}
& \underset{\widehat{\mathcal{G}}, \widehat{\boldsymbol{U}}, \widehat{\boldsymbol{V}}, \widehat{\boldsymbol{W}}}{\operatorname{minimize}} f_{T}(\widehat{\mathcal{G}}, \widehat{\boldsymbol{U}}, \widehat{\boldsymbol{V}}, \widehat{\boldsymbol{W}}), \quad \text { where } \\
& f_{T}(\widehat{\boldsymbol{U}}, \widehat{\boldsymbol{V}}, \widehat{\boldsymbol{W}}, \widehat{\mathcal{G}})=\left\|\mathcal{Y}_{H}-\llbracket \widehat{\mathcal{G}} ; \boldsymbol{P}_{\mathbf{1}} \widehat{\boldsymbol{U}}, \boldsymbol{P}_{\mathbf{2}} \widehat{\boldsymbol{V}}, \widehat{\boldsymbol{W}} \rrbracket\right\|_{F}^{2} \\
& +\lambda\left\|\mathcal{Y}_{M}-\llbracket \widehat{\mathcal{G}} ; \widehat{\boldsymbol{U}}, \widehat{\boldsymbol{V}}, \boldsymbol{P}_{\boldsymbol{M}} \widehat{\boldsymbol{W}} \rrbracket\right\|_{F}^{2} .
\end{aligned}
$$

Rather than finding a (local) minimum of (5), we propose two (semi-algebraic) closed-form solutions that are suboptimal, but are fast and easy to calculate.

\section{B. An SVD-based algorithm for known spatial degradation}

A two-stage approach inspired by the high-order SVD (HOSVD) [12] consists in:

- using the truncated SVD of MSI and HSI to obtain the factors $\widehat{U}, \widehat{V}, \widehat{W}$ in (4);

- performing the data fusion by minimizing the objective (6) only with respect to the core tensor $\widehat{\mathcal{G}}$.

This method, called SCOTT, is given in Algorithm 5

Note that, under conditions provided in Section $\mathrm{V}$, Algorithm 5 gives a solution to the algebraic decomposition problem (4) in the noise-free case $\left(\mathcal{E}_{M}=\mathbf{0}, \mathcal{E}_{H}=\mathbf{0}\right)$.

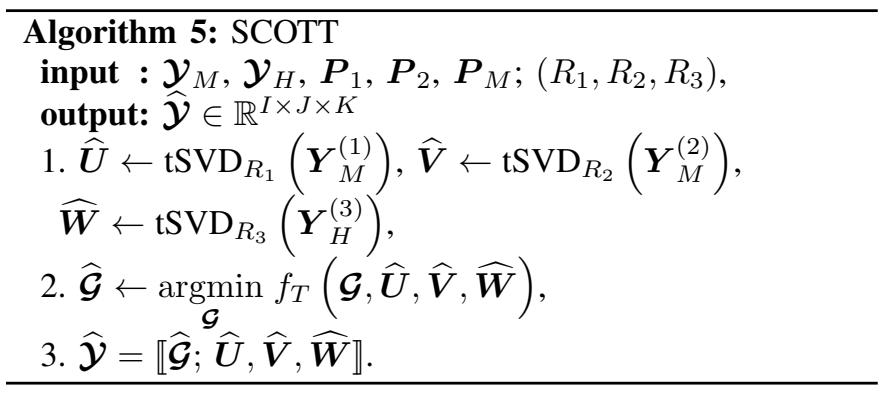

${ }^{3}$ Note that another method based on Tucker factorization and sparse approximation was proposed in [21]. However, no recoverability condition is available for that method.

${ }^{4}$ Note that our final goal is not parameter estimation, but rather the recovery of the SRI tensor. We use the word "estimating" just to underscore that it is not necessary to formulate the recovery problem as an optimization problem.
Step 2 of Algorithm 5 is the least squares problem

$$
\underbrace{\left[\begin{array}{c}
\widehat{\boldsymbol{W}} \otimes \boldsymbol{P}_{2} \widehat{\boldsymbol{V}} \otimes \boldsymbol{P}_{1} \widehat{\boldsymbol{U}} \\
\sqrt{\lambda} \boldsymbol{P}_{M} \widehat{\boldsymbol{W}} \otimes \widehat{\boldsymbol{V}} \otimes \widehat{\boldsymbol{U}}
\end{array}\right]}_{\boldsymbol{X}} \operatorname{vec}\{\widehat{\mathcal{G}}\} \approx \underbrace{\left[\begin{array}{c}
\operatorname{vec}\left\{\mathcal{Y}_{H}\right\} \\
\sqrt{\lambda} \operatorname{vec}\left\{\mathcal{Y}_{M}\right\}
\end{array}\right]}_{\boldsymbol{z}}
$$

that can be solved through normal equations of the form

$$
\left(\boldsymbol{X}^{\top} \boldsymbol{X}\right) \operatorname{vec}\{\widehat{\mathcal{G}}\}=\boldsymbol{X}^{\top} \boldsymbol{z}
$$

The matrix on the left-hand side of (7) can be written as

$$
\begin{aligned}
\boldsymbol{X}^{\top} \boldsymbol{X}=\boldsymbol{I}_{R_{3}} \otimes & \left(\widehat{\boldsymbol{V}}^{\top} \boldsymbol{P}_{2}^{\top} \boldsymbol{P}_{2} \widehat{\boldsymbol{V}}\right) \otimes\left(\widehat{\boldsymbol{U}}^{\top} \boldsymbol{P}_{1}^{\top} \boldsymbol{P}_{1} \widehat{\boldsymbol{U}}\right) \\
& +\lambda\left(\widehat{\boldsymbol{W}}^{\top} \boldsymbol{P}_{M}^{\top} \boldsymbol{P}_{M} \widehat{\boldsymbol{W}}\right) \otimes \boldsymbol{I}_{R_{1} R_{2}},
\end{aligned}
$$

and the vector on the right-hand side is

$$
\begin{aligned}
\boldsymbol{X}^{\top} \boldsymbol{z} & =\operatorname{vec}\left\{\llbracket \mathcal{Y}_{H} ; \widehat{\boldsymbol{U}}^{\top} \boldsymbol{P}_{1}^{\top}, \widehat{\boldsymbol{V}}^{\top} \boldsymbol{P}_{2}^{\top}, \widehat{\boldsymbol{W}}^{\top} \rrbracket\right\} \\
& +\lambda \operatorname{vec}\left\{\llbracket \mathcal{Y}_{M} ; \widehat{\boldsymbol{U}}^{\top}, \widehat{\boldsymbol{V}}^{\top}, \widehat{\boldsymbol{W}}^{\top} \boldsymbol{P}_{M}^{\top} \rrbracket\right\} .
\end{aligned}
$$

The normal equations can be viewed as a (generalized) Sylvester equation and (as in the case of STEREO) efficient solvers can be used (see Appendix A for more details). Thus the total cost of SCOTT algorithm becomes

- $O\left(\min \left(R_{1}, R_{2}\right) I J K_{M}+R_{3} I_{H} J_{H} K\right)$ flops for computing the truncated SVDs and computing $\boldsymbol{X}^{\top} \boldsymbol{z}$;

- $O\left(\min \left(R_{3}^{3}+\left(R_{1} R_{2}\right)^{3}, R_{1}^{3}+\left(R_{2} R_{3}\right)^{3}\right)\right)$ flops for solving the Sylvester equation.

It is easy to see that the computational complexity of SCOTT is comparable to that of one iteration of STEREO and can be smaller if the multilinear ranks are small.

\section{An algorithm for unknown spatial degradation}

In this subsection, we show that is also possible to develop a blind SVD-based algorithm, in the same spirit as Algorithm 3 The algorithm does not need knowledge of $\boldsymbol{P}_{1}, \boldsymbol{P}_{2}$ and is based on the HOSVD of the MSI tensor.

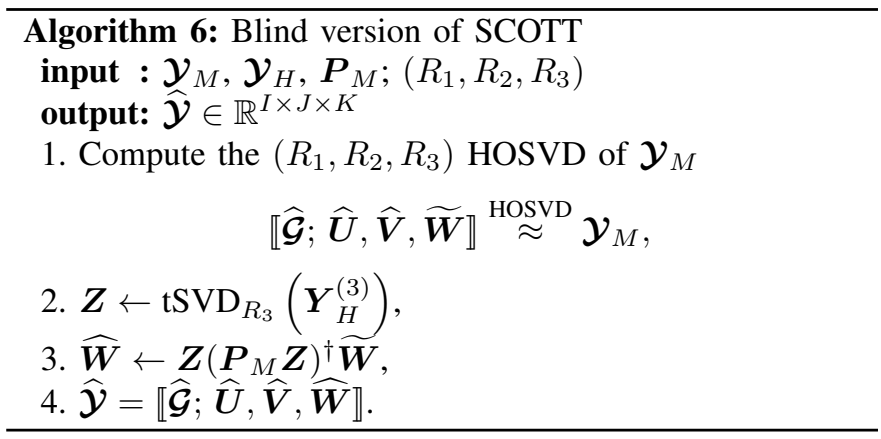

The total computational complexity of Algorithm 6 is

$$
O\left(\min \left(R_{1}, R_{2}\right) I J K_{M}+R_{3} I_{H} J_{H} K\right) \text { flops }
$$

and is dominated by the cost of the truncated SVD, because step 3 is very cheap. However, a specific drawback of Algorithm 7, similarly to Algorithm 3 , is that $R_{3}$ should not exceed $K_{M}$, since the multilinear rank is employed in the HOSVD of subblocks of $\mathcal{Y}_{M}$. 
Finally, similarly to SCUBA, we can use a block version of Algorithm 6, which we call B-SCOTT (which stands for "Blind SCOTT"). There is no confusion, as Algorithm 6 is a special case of Algorithm 7 where the degraded image cubes are not split into blocks.

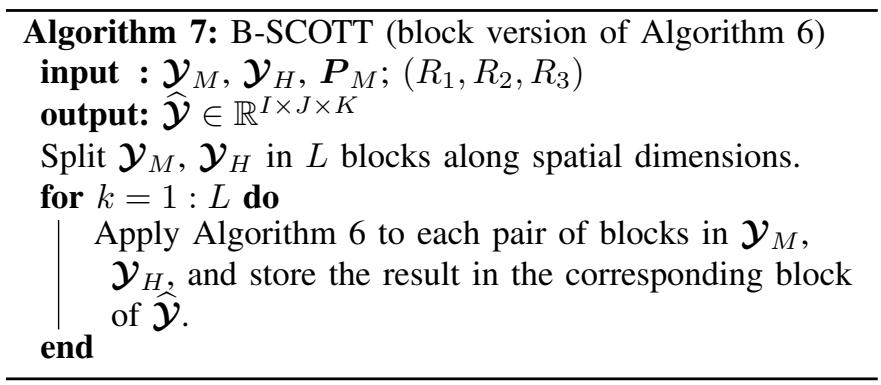

\section{RECOVERABILITY OF THE TUCKER MODEL}

In this section, we establish conditions for correct SRI tensor recovery in the coupled Tucker model. The proof of such conditions for the $\mathrm{CP}$ model in [9] relied on the uniqueness (identifiability) property of the CPD of the MSI. We show that, although the Tucker decomposition is not unique, the correct recovery is still possible. Moreover, we prove that in some cases where the CPD in [9] is not unique, the SRI tensor is still uniquely recovered using the $\mathrm{CP}$ model.

\section{A. Deterministic exact recovery conditions}

We begin with a deterministic result on recoverability 5

Theorem V.1. Let a Tucker decomposition of $\mathcal{Y}$ be

$$
\mathcal{Y}=\llbracket \mathcal{G} ; \boldsymbol{U}, \boldsymbol{V}, \boldsymbol{W} \rrbracket
$$

where $\mathcal{G} \in \mathbb{R}^{R_{1} \times R_{2} \times R_{3}}$, and $\boldsymbol{U} \in \mathbb{R}^{I \times R_{1}}, \boldsymbol{V} \in \mathbb{R}^{J \times R_{2}}$, $\boldsymbol{W} \in \mathbb{R}^{K \times R_{3}}$ have full column rank. We also assume that $\mathcal{E}_{M}, \mathcal{E}_{H}=\mathbf{0}$ in (2).

1) If

$$
\begin{aligned}
& \operatorname{rank}\left\{\boldsymbol{Y}_{M}^{(1)}\right\}=R_{1}, \operatorname{rank}\left\{\boldsymbol{Y}_{M}^{(2)}\right\}=R_{2}, \\
& \operatorname{rank}\left\{\boldsymbol{Y}_{H}^{(3)}\right\}=R_{3},
\end{aligned}
$$

and one of the following conditions holds true:

a) either $\operatorname{rank}\left\{\boldsymbol{P}_{1} \boldsymbol{U}\right\}=R_{1}$ and $\operatorname{rank}\left\{\boldsymbol{P}_{2} \boldsymbol{V}\right\}=R_{2}$;

b) or $\operatorname{rank}\left\{\boldsymbol{P}_{M} \boldsymbol{W}\right\}=R_{3}$.

Then there exists only one $\widehat{\mathcal{Y}}$ with multilinear rank at most $\left(R_{1}, R_{2}, R_{3}\right)$ such that $\widehat{\mathcal{Y}} \bullet_{3} \boldsymbol{P}_{M}=\mathcal{Y}_{M}$ and $\widehat{\mathcal{Y}} \bullet_{1} \boldsymbol{P}_{1} \bullet_{2} \boldsymbol{P}_{2}=\mathcal{Y}_{H}$.

2) If $\boldsymbol{U}, \boldsymbol{V}, \boldsymbol{W}$ none of the conditions a) and b) are satisfied, then there exist infinitely many $\widehat{\mathcal{Y}}$ of the form

$$
\begin{aligned}
& \widehat{\mathcal{Y}}=\llbracket \widehat{\mathcal{G}} ; \widehat{\boldsymbol{U}}, \widehat{\boldsymbol{V}}, \widehat{\boldsymbol{W}} \rrbracket, \\
& \widehat{\boldsymbol{U}} \in \mathbb{R}^{I \times R_{1}}, \widehat{\boldsymbol{V}} \in \mathbb{R}^{J \times R_{2}}, \widehat{\boldsymbol{W}} \in \mathbb{R}^{K \times R_{3}},
\end{aligned}
$$

such that $\widehat{\mathcal{Y}} \bullet_{3} \boldsymbol{P}_{M}=\mathcal{Y}_{M}$ and $\widehat{\mathcal{Y}} \bullet_{1} \boldsymbol{P}_{1} \bullet_{2} \boldsymbol{P}_{2}=\mathcal{Y}_{H}$; in fact, $\|\widehat{\mathcal{Y}}-\mathcal{Y}\|$ can be arbitrary large for such $\widehat{\mathcal{Y}}$.

\footnotetext{
${ }^{5}$ In this paper, we prefer to use the term "recoverability of the SRI" rather than "identifiability of the SRI" used in [9], in order to avoid confusion with identifiability of the low-rank model.
}

Proof. First of all, we note that by [22, Theorem 13.16], the singular values of the matrix $\boldsymbol{X}^{\top} \boldsymbol{X}=\boldsymbol{I}_{R_{3}} \otimes \boldsymbol{A}+\boldsymbol{D} \otimes \boldsymbol{I}_{R_{1} R_{2}}$ in (8) are all sums of the pairs of eigenvalues of

$$
\underbrace{\left(\widehat{\boldsymbol{V}}^{\top} \boldsymbol{P}_{2}^{\top} \boldsymbol{P}_{2} \widehat{\boldsymbol{V}}\right) \otimes\left(\widehat{\boldsymbol{U}}^{\top} \boldsymbol{P}_{1}^{\top} \boldsymbol{P}_{1} \widehat{\boldsymbol{U}}\right)}_{\boldsymbol{A}}, \underbrace{\lambda \widehat{\boldsymbol{W}}^{\top} \boldsymbol{P}_{M}^{\top} \boldsymbol{P}_{M} \widehat{\boldsymbol{W}}}_{\boldsymbol{D}} .
$$

We also assume without loss of generality that $\boldsymbol{U}, \boldsymbol{V}, \boldsymbol{W}$ have orthonormal columns.

- Proof of 2) Assume that $\operatorname{rank}\left\{\boldsymbol{P}_{1} \boldsymbol{U}\right\} \operatorname{rank}\left\{\boldsymbol{P}_{2} \boldsymbol{U}\right\}<$ $R_{1} R_{2}$ and $\operatorname{rank}\left\{\boldsymbol{P}_{M} \boldsymbol{W}\right\}<R_{3}$. If we set $\widehat{\boldsymbol{U}}=\boldsymbol{U}$, $\widehat{\boldsymbol{V}}=\boldsymbol{V}, \widehat{\boldsymbol{W}}=\boldsymbol{W}$, then $\operatorname{rank}\{\boldsymbol{A}\}<R_{1} R_{2}, \operatorname{rank}\{\boldsymbol{D}\}<$ $R_{3}$ and $\operatorname{rank}\left\{\boldsymbol{X}^{\top} \boldsymbol{X}\right\}<R_{1} R_{2} R_{3}$. Therefore the system (7) is underdetermined, and there is an infinite number of solutions $\widehat{\mathcal{G}} \in \mathbb{R}^{R_{1} \times R_{2} \times R_{3}}$. Note that if we define $\widehat{\mathcal{Y}}=\llbracket \widehat{\mathcal{G}} ; \boldsymbol{U}, \boldsymbol{V}, \boldsymbol{W} \rrbracket$, then it is an admissible solution, i.e., $\widehat{\mathcal{Y}} \bullet_{3} \boldsymbol{P}_{M}=\mathcal{Y}_{M}$ and $\widehat{\mathcal{Y}} \bullet_{1} \boldsymbol{P}_{1} \bullet_{2} \boldsymbol{P}_{2}=\mathcal{Y}_{H}$. On the other hand, due to orthogonality of the bases, $\|\widehat{\mathcal{Y}}-\mathcal{Y}\|_{F}=\|\widehat{\mathcal{G}}-\mathcal{G}\|_{F}$, which can be made arbitrary large due to nonuniqueness of the solution to (7).

- Proof of 1) Let us choose $\widehat{\boldsymbol{U}} \in \mathbb{R}^{I \times R_{1}}, \widehat{\boldsymbol{V}} \in \mathbb{R}^{J \times R_{2}}$, and $\widehat{W} \in \mathbb{R}^{K \times R_{3}}$ to be orthogonal bases of the row spaces of $\boldsymbol{Y}_{M}^{(1)}, \boldsymbol{Y}_{M}^{(2)}$ and $\boldsymbol{Y}_{H}^{(3)}$ respectively. First, by (11), the rank of unfoldings does not drop after degradation, hence

$$
\widehat{U}=U Q_{U}, \widehat{V}=V Q_{V}, \widehat{W}=W Q_{W},
$$

where $Q_{U}, Q_{V}, Q_{W}$ are some rotation matrices. Next, due to conditions on the ranks of $\boldsymbol{P}_{1} \boldsymbol{U}, \boldsymbol{P}_{2} \boldsymbol{U}$ and $\boldsymbol{P}_{M} \boldsymbol{W}$, we get that $\operatorname{rank}\left\{\boldsymbol{X}^{\top} \boldsymbol{X}\right\}=R_{1} R_{2} R_{3}$ because of 12 . Hence the solution $\widehat{\mathcal{G}}$ of (7) is unique. Finally, we note that the reconstructed tensor can be expressed as

$$
\operatorname{vec}\{\widehat{\mathcal{Y}}\}=(\widehat{\boldsymbol{W}} \otimes \widehat{\boldsymbol{V}} \otimes \widehat{\boldsymbol{U}})\left(\boldsymbol{X}^{\top} \boldsymbol{X}\right)^{-1} \boldsymbol{X}^{\top} \boldsymbol{z},
$$

where the right-hand side does not depend on the rotation matrices $\boldsymbol{Q}_{U}, \boldsymbol{Q}_{V}$, and $\boldsymbol{Q}_{W}$ due to the definition of $\boldsymbol{X}$. Hence, the reconstructed tensor $\widehat{\mathcal{Y}}$ is unique.

Corollary V.2. If the conditions of Theorem V.1 (part 1) hold, then any minimizer of (5) recovers $\mathcal{Y}$, i.e.

$$
\mathcal{Y}=\llbracket \widehat{\mathcal{G}} ; \widehat{\boldsymbol{U}}, \widehat{\boldsymbol{V}}, \widehat{\boldsymbol{W}} \rrbracket .
$$

In addition, Algorithm 5 recovers $\mathcal{Y}$ for all cases of recoverability in Theorem V.1.

The recoverability results derived in Theorem V.1 are valid if a Tucker decomposition is used, and if its core tensor is dense. But they still remain valid if the core tensor is diagonal or block diagonal. For this reason, they also apply to CPD or BTD decompositions if the tensor rank is smaller than dimensions. In particular, recoverability can be ensured under mild conditions when the CPD is not unique, e.g. in the presence of collinear factors, as shown in the following corollary.

Corollary V.3 (Recoverability for CPD model with partial uniqueness). Assume that the SRI has a CPD $\mathcal{Y}=\llbracket \boldsymbol{A}, \boldsymbol{B}, \boldsymbol{C} \rrbracket$ of $\operatorname{rank} F \leq \min \left(I_{H}, J_{H}\right)$, such that

$\operatorname{rank}\{\boldsymbol{A}\}=\operatorname{rank}\left\{\boldsymbol{P}_{1} \boldsymbol{A}\right\}=\operatorname{rank}\{\boldsymbol{B}\}=\operatorname{rank}\left\{\boldsymbol{P}_{2} \boldsymbol{B}\right\}=F$ 
and $\boldsymbol{P}_{M} \boldsymbol{C}$ does not have zero columns. We also assume that $\mathcal{E}_{M}, \mathcal{E}_{H}=\mathbf{0}$ in (2). Then any minimizer of (3) recovers $\mathcal{Y}$.

Proof. Since the original factors $A, B, C$ yield zero error in (3), hence any global minimizer $(\widehat{\boldsymbol{A}}, \widehat{\boldsymbol{B}}, \widehat{\boldsymbol{C}})$ of $(3)$, satisfies

$$
\llbracket \boldsymbol{P}_{1} \widehat{\boldsymbol{A}}, \boldsymbol{P}_{2} \widehat{\boldsymbol{B}}, \widehat{\boldsymbol{C}} \rrbracket=\mathcal{Y}_{H} \text { and } \llbracket \widehat{\boldsymbol{A}}, \widehat{\boldsymbol{B}}, \boldsymbol{P}_{M} \widehat{\boldsymbol{C}} \rrbracket=\mathcal{Y}_{M} .
$$

Due to the conditions of the corollary, $\llbracket \boldsymbol{P}_{1} \boldsymbol{A}, \boldsymbol{P}_{2} \boldsymbol{B}, \boldsymbol{C} \rrbracket$ and $\llbracket \boldsymbol{A}, \boldsymbol{B}, \boldsymbol{P}_{M} \boldsymbol{C} \rrbracket$ satisfy partial uniqueness conditions in $\llbracket 23$, Theorem 2.2]. Hence (after permutations and rescaling of factors), we have $\widehat{\boldsymbol{C}}=\boldsymbol{C}$ and

$$
\operatorname{rank}\{\boldsymbol{C}\}=\operatorname{rank}\{\widehat{\boldsymbol{C}}\}=\operatorname{rank}\left\{\boldsymbol{Y}_{H}^{(3)}\right\}=R_{3} .
$$

Moreover, since

$$
\boldsymbol{Y}_{M}^{(1)}=\left(\boldsymbol{P}_{M} \boldsymbol{C} \odot \boldsymbol{B}\right) \boldsymbol{A}^{\top}, \quad \boldsymbol{Y}_{M}^{(2)}=\left(\boldsymbol{P}_{M} \boldsymbol{C} \odot \boldsymbol{A}\right) \boldsymbol{B}^{\top},
$$

and $\boldsymbol{P}_{M} \boldsymbol{C}$ does not have zero columns, we have that

$$
\begin{aligned}
& \operatorname{rank}\{\boldsymbol{A}\}=\operatorname{rank}\{\widehat{\boldsymbol{A}}\}=\operatorname{rank}\left\{\boldsymbol{Y}_{M}^{(1)}\right\}=R_{1}=F, \\
& \operatorname{rank}\{\boldsymbol{B}\}=\operatorname{rank}\{\widehat{\boldsymbol{B}}\}=\operatorname{rank}\left\{\boldsymbol{Y}_{M}^{(2)}\right\}=R_{2}=F .
\end{aligned}
$$

Therefore, both $(\boldsymbol{A}, \boldsymbol{B}, \boldsymbol{C})$ and $(\widehat{\boldsymbol{A}}, \widehat{\boldsymbol{B}}, \widehat{\boldsymbol{C}})$ are particular solutions of Problem (5) with an additional constraint that the tensor rank of $\mathcal{G}$ is at most $F$. Since, by Theorem V.1 any solution of (5) recovers $\mathcal{Y}$ uniquely, the proof is complete.

Note that the conditions of Corollary V.3 are quite restrictive for real applications. But, they probably can be relaxed by using Kruskal ranks and a more general formulation in [23. Theorem 2.1] (see also [24]).

\section{B. Exact recoverability for generic tensors}

From the deterministic recovery conditions, we can establish the generic recoverability results.

Theorem V.4. Assume that $\boldsymbol{P}_{1} \in \mathbb{R}^{I_{H} \times I}, \boldsymbol{P}_{2} \in \mathbb{R}^{J_{H} \times J}$, and $\boldsymbol{P}_{M} \in \mathbb{R}^{K_{M} \times K}$ are fixed full row-rank matrices. Let $\mathcal{Y}$ have decomposition (10), where $R_{1} \leq I, R_{2} \leq J, R_{3} \leq K$, and $\mathcal{G} \in \mathbb{R}^{R_{1} \times R_{2} \times R_{3}}, \boldsymbol{U} \in \mathbb{R}^{I \times R_{1}}, \boldsymbol{V} \in \mathbb{R}^{J \times R_{2}}, \boldsymbol{W} \in \mathbb{R}^{K \times R_{3}}$ are random tensors and matrices, distributed according to an absolutely continuous probability distribution. We also assume that $\mathcal{E}_{M}, \mathcal{E}_{H}=\mathbf{0}$ in (2).

1) If $R_{3} \leq K_{M}$ or $\left(R_{1}, R_{2}\right) \leq\left(I_{H}, J_{H}\right)$ and

$$
\left\{\begin{array}{l}
R_{1} \leq \min \left(R_{3}, K_{M}\right) R_{2}, \\
R_{2} \leq \min \left(R_{3}, K_{M}\right) R_{1}, \\
R_{3} \leq \min \left(R_{1}, I_{H}\right) \min \left(R_{2}, J_{H}\right),
\end{array}\right.
$$

then with probability 1 there exists a unique tensor $\widehat{\mathcal{Y}}$ with multilinear rank at most $\left(R_{1}, R_{2}, R_{3}\right)$ such that $\widehat{\mathcal{Y}} \bullet_{3} \boldsymbol{P}_{M}=\mathcal{Y}_{M}$ and $\hat{\mathcal{Y}} \bullet_{1} \boldsymbol{P}_{1} \bullet_{2} \boldsymbol{P}_{2}=\mathcal{Y}_{H}$.

2) If $R_{3}>K_{M}$ and $\left(R_{1}>I_{H}\right.$ or $\left.R_{2}>J_{H}\right)$, then with probability 1 the reconstruction is non-unique, i.e. there exist infinitely many $\widehat{\mathcal{Y}}$ of the form

$$
\begin{aligned}
& \widehat{\mathcal{Y}}=\llbracket \widehat{\mathcal{G}} ; \widehat{\boldsymbol{U}}, \widehat{\boldsymbol{V}}, \widehat{\boldsymbol{W}} \rrbracket, \\
& \widehat{\boldsymbol{U}} \in \mathbb{R}^{I \times R_{1}}, \widehat{\boldsymbol{V}} \in \mathbb{R}^{J \times R_{2}}, \widehat{\boldsymbol{W}} \in \mathbb{R}^{K \times R_{3}},
\end{aligned}
$$

such that $\widehat{\mathcal{Y}} \bullet_{3} \boldsymbol{P}_{M}=\mathcal{Y}_{M}$ and $\widehat{\mathcal{Y}} \bullet_{1} \boldsymbol{P}_{1} \bullet_{2} \boldsymbol{P}_{2}=\mathcal{Y}_{H}$; in fact, $\|\widehat{\mathcal{Y}}-\mathcal{Y}\|$ can be arbitrary large for such $\widehat{\mathcal{Y}}$.
Proof. - Proof of 2) follows from Theorem V.1 (part 2)

- Proof of 1) First, without loss of generality, we can replace $\boldsymbol{P}_{1}, \boldsymbol{P}_{2}, \boldsymbol{P}_{M}$ with the following of same size:

$$
\widetilde{\boldsymbol{P}}_{1}=\left[\begin{array}{c}
\boldsymbol{I}_{I_{H}} \\
\mathbf{0}
\end{array}\right]^{\top}, \widetilde{\boldsymbol{P}}_{2}=\left[\begin{array}{c}
\boldsymbol{I}_{J_{H}} \\
\mathbf{0}
\end{array}\right]^{\top}, \widetilde{\boldsymbol{P}}_{M}=\left[\begin{array}{c}
\boldsymbol{I}_{K_{M}} \\
\mathbf{0}
\end{array}\right]^{\top} .
$$

Indeed, let us explain why it is so, for example for $\boldsymbol{P}_{1} \in$ $\mathbb{R}^{I_{H} \times I}$. There exists a nonsingular matrix 6 such that

$$
\boldsymbol{P}_{1} \boldsymbol{T}=\left[\begin{array}{ll}
\boldsymbol{I}_{I_{H}} & \mathbf{0}
\end{array}\right] .
$$

If we take $\widetilde{\boldsymbol{U}}=\boldsymbol{T}^{-1} \boldsymbol{U}$ then $\boldsymbol{P}_{1} \boldsymbol{U}=\widetilde{\boldsymbol{P}}_{1} \widetilde{\boldsymbol{U}}$. Note that a nonsingular transformation preserves absolute continuity of the distribution; hence $\boldsymbol{U}$ has an absolutely continuous distribution if and only if $\widetilde{\boldsymbol{U}}$ has one.

Therefore, under the assumptions on distribution of $\boldsymbol{U}$, $\boldsymbol{V}, \boldsymbol{W}$ the following implications hold with probability 1

$$
\begin{aligned}
& R_{1} \leq I_{H} \Rightarrow \operatorname{rank}\left\{\boldsymbol{U}_{1: I_{H},:}\right\}=R_{1}, \\
& R_{2} \leq J_{H} \Rightarrow \operatorname{rank}\left\{\boldsymbol{V}_{1: J_{H},:}\right\}=R_{2}, \\
& R_{3} \leq K_{M} \Rightarrow \operatorname{rank}\left\{\boldsymbol{W}_{1: K_{M},:}\right\}=R_{3} .
\end{aligned}
$$

Next, we are going to show how the other set of conditions imply (11). We will prove it only for the first condition (the others are analogous).

Note that the first unfolding can be written as

$$
\boldsymbol{Y}_{M}^{(1)}=\left(\boldsymbol{W}_{1: K_{M},:} \otimes \boldsymbol{V}\right) \boldsymbol{G}^{(1)} \boldsymbol{U}^{\top} .
$$

Due to the dimensions of the terms in the product, this matrix is at most rank $R_{1}$. Due to semicontinuity of the rank function, $\boldsymbol{Y}_{H}^{(1)}$ will be generically of rank $R_{1}$ if we can provide just a single example of $\boldsymbol{U}, \boldsymbol{V}, \boldsymbol{W}, \mathcal{G}$, achieving the condition $\operatorname{rank}\left\{\boldsymbol{Y}_{M}^{(1)}\right\}=R_{1}$. Indeed, if $R_{1} \leq \min \left(R_{3}, K_{M}\right) R_{2}$, such an example is given by

$\boldsymbol{U}=\left[\begin{array}{c}\boldsymbol{I}_{R_{1}} \\ \mathbf{0}\end{array}\right], \boldsymbol{V}=\left[\begin{array}{c}\boldsymbol{I}_{R_{2}} \\ \mathbf{0}\end{array}\right], \boldsymbol{W}=\left[\begin{array}{c}\boldsymbol{I}_{R_{3}} \\ \mathbf{0}\end{array}\right], \boldsymbol{G}^{(1)}=\left[\begin{array}{c}\boldsymbol{I}_{R_{1}} \\ \mathbf{0}\end{array}\right]$,

which completes the proof.

We illustrate the statement of Theorem $\mathrm{V} .4$ for the case $I=J, I_{H}=J_{H}$ and $R_{1}=R_{2}$. In Figure 1 we show that the space of parameters $\left(R_{1}, R_{3}\right)$ is split into two regions: recoverable and non-recoverable. The hatched area corresponds to the parameters where condition (13) is not satisfied.

Remark V.5. In the proof of Theorem V.4 it was shown that we can assume that the degradation operators are given in [14. In that case, the degraded tensors $\mathcal{Y}_{M}$ and $\mathcal{Y}_{H}$ are just the subtensors (slabs) i.e.

$$
\mathcal{Y}_{M}=\mathcal{Y}_{:,,, 1: K_{M}}, \quad \mathcal{Y}_{H}=\mathcal{Y}_{1: I_{H}, 1: J_{H},:} .
$$

Hence the recoverability of Tucker super-resolution model is equivalent to uniqueness of tensor completion [25], that is the recovery of $\mathcal{Y}$ from known subtensors $\mathcal{Y}_{:,:, 1: K_{M}}$ and $\mathcal{Y}_{1: I_{H}, 1: J_{H},:}$, shown in Figure 2

We note that for the coupled CP approach (STEREO), the connection with tensor completion was recently used in [26] for accelerated reconstruction of fMRI images.

\footnotetext{
${ }^{6}$ For example, $\boldsymbol{T}=\left[\boldsymbol{P}_{1}^{\dagger} \boldsymbol{F}\right]$, where $\boldsymbol{F} \in \mathbb{R}^{I \times\left(I-I_{H}\right)}, \boldsymbol{P}_{1} \boldsymbol{F}=\mathbf{0}$.
} 


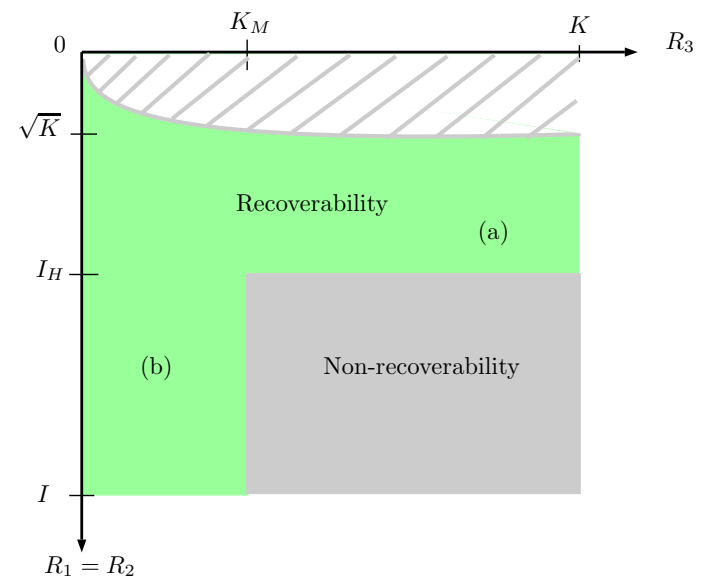

Fig. 1. Identifiability region depending on $R_{1}$ and $R_{3}$

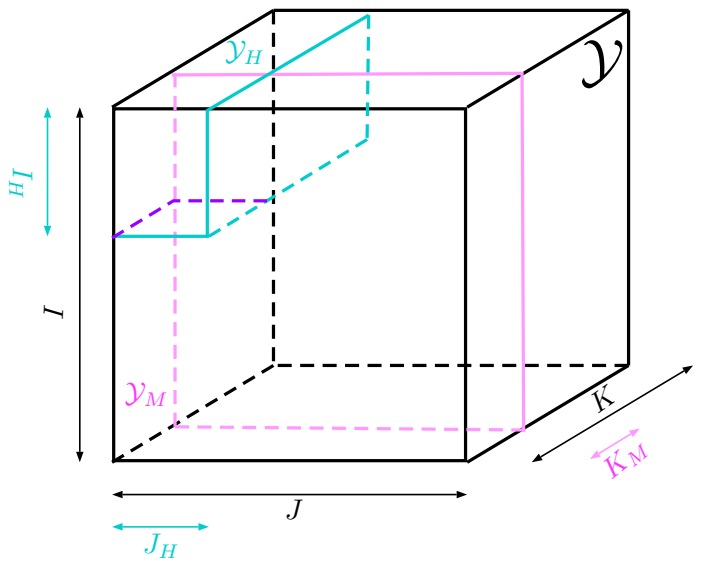

Fig. 2. Recovery of $\mathcal{Y}$ from $\mathcal{Y}_{:,:, 1: K_{M}}$ (pink), $\mathcal{Y}_{1: I_{H}, 1: J_{H},:}$ (blue)

\section{Recoverability in the blind case}

Similarly to Theorem V.1, we can prove correct recovery for Algorithm 6 under relaxed degradation model. We assume that the MSI is degraded as before, and HSI is degraded slicewise by an unknown linear operator $\mathcal{P}_{s}: \mathbb{R}^{I \times J} \rightarrow \mathbb{R}^{I_{H} \times J_{H}}$.

$$
\begin{cases}\mathcal{Y}_{M} & =\mathcal{Y} \bullet_{3} \boldsymbol{P}_{M}, \\ \left(\mathcal{Y}_{H}\right)_{:,:, k} & =\mathcal{P}_{s}\left(\mathcal{Y}_{:,,, k}\right) .\end{cases}
$$

Then it is easy to prove the following analogue of Theorem V.1

Proposition V.6. Let $\mathcal{Y}$ have a Tucker decomposition

$$
\mathcal{Y}=\llbracket \mathcal{G} ; \boldsymbol{U}, \boldsymbol{V}, \boldsymbol{W} \rrbracket,
$$

where $\mathcal{G} \in \mathbb{R}^{R_{1} \times R_{2} \times R_{3}}$, and $\boldsymbol{U} \in \mathbb{R}^{I \times R_{1}}, \boldsymbol{V} \in \mathbb{R}^{J \times R_{2}}$, $\boldsymbol{W} \in \mathbb{R}^{K \times R_{3}}$ are full column rank.

If $\operatorname{rank}\left\{\boldsymbol{Y}_{H}^{(3)}\right\}=R_{3}$ and $\operatorname{rank}\left\{\boldsymbol{P}_{M} \boldsymbol{W}\right\}=R_{3}$, then Algorithm 6 recovers $\mathcal{Y}$ correctly.

Proof. Indeed, $\mathcal{Y}_{M}=\llbracket \mathcal{G} ; \boldsymbol{U}, \boldsymbol{V}, \boldsymbol{P}_{M} \boldsymbol{W} \rrbracket$. Therefore, since $\operatorname{rank}\left\{\boldsymbol{P}_{M} \boldsymbol{W}\right\}=R_{3}$, the multilinear rank of $\mathcal{Y}_{M}$ is equal to the one of $\mathcal{Y}$ and

$$
\mathcal{Y}=\mathcal{Y}_{M} \ddot{3}_{3}\left(\boldsymbol{W}\left(\boldsymbol{P}_{M} \boldsymbol{W}\right)^{\dagger}\right)
$$

Finally, due to the condition $\operatorname{rank}\left\{\boldsymbol{Y}_{H}^{(3)}\right\}=R_{3}$, step 2 of Algorithm 6 recovers $\boldsymbol{W}$ up to a change of basis, i.e., $\boldsymbol{Z}=$ $\boldsymbol{W} \boldsymbol{O}$, where $\boldsymbol{O} \in \mathbb{R}^{R_{3} \times R_{3}}$ is an orthogonal matrix. Finally, due to the properties of the pseudoinverse

$$
\left(\boldsymbol{W} \boldsymbol{O}\left(\boldsymbol{P}_{M} \boldsymbol{W} \boldsymbol{O}\right)^{\dagger}\right)=\boldsymbol{W}\left(\boldsymbol{P}_{M} \boldsymbol{W}\right)^{\dagger},
$$

which completes the proof.

\section{NUMERICAL EXPERIMENTS}

All simulations were run on a MacBook Pro with $2.3 \mathrm{GHz}$ Intel Core i5 and 16GB RAM. The code is implemented in MATLAB. For basic tensor operations we used TensorLab 3.0 [27]. The results are reproducible and the codes are available online at https://github.com/cprevost4/HSR_Software.

\section{A. Degradation model}

Experiments are conducted on a set of semi-real and synthetic examples, in which the groundtruth SRI is artificially degraded to $\mathcal{Y}_{H}$ and $\mathcal{Y}_{M}$ by the degradation matrices $\boldsymbol{P}_{1}$, $\boldsymbol{P}_{2}$ and $\boldsymbol{P}_{M}$ according to model (2).

For spatial degradation, we follow the commonly used Wald's protocol [17]. The matrices $\boldsymbol{P}_{1}, \boldsymbol{P}_{2}$ are computed with a separable Gaussian blurring kernel of size $q=9$. Then, downsampling is performed along each spatial dimension with a ratio $d=4$ between $I, J$ and $I_{H}, J_{H}$, as in [9]. We refer to Appendix $B$ for more details on the construction of $\boldsymbol{P}_{1}, \boldsymbol{P}_{2}$.

In this paper, we consider two spectral responses used to generate the spectral degradation matrix $\boldsymbol{P}_{M}$. In all the semi-real examples, available online at [28], the bands corresponding to water absorption are first removed as in [9]. The LANDSAT sensor spans the spectrum from $400 \mathrm{~nm}$ to $2500 \mathrm{~nm}$ for the HSI and produces a 6-band MSI corresponding to wavelengths $450-520 \mathrm{~nm}$ (blue), 520-600nm (green), 630$690 \mathrm{~nm}$ (red), $760-900 \mathrm{~nm}$ (near-IR), $1550-1750 \mathrm{~nm}$ (shortwaveIR) and 2050-2350nm (shortwave-IR2). The second response corresponds to a QuickBird sensor, which spans the spectrum from $430 \mathrm{~nm}$ to $860 \mathrm{~nm}$ for the HSI and produces a 4-band MSI which bands correspond to wavelengths $430-545 \mathrm{~nm}$ (blue), 466-620nm (green), 590-710nm (red) and 715-918nm (nearIR). The spectral degradation matrix $\boldsymbol{P}_{M}$ is a selectionaveraging matrix that selects the common spectral bands of the SRI and MSI.

\section{B. Metrics}

As for the experimental setup, we follow [9]; we compare the groundtruth SRI with the recovered SRI obtained by the algorithms. The main performance metric used in comparisons is reconstruction Signal-to-Noise ratio (R-SNR) used in [3]:

$$
\mathrm{R}-\mathrm{SNR}=10 \log _{10}\left(\frac{\|\mathcal{Y}\|_{F}^{2}}{\|\widehat{\mathcal{Y}}-\mathcal{Y}\|_{F}^{2}}\right) .
$$

In addition to R-SNR, we consider different metrics from [3] described below:

$$
\mathrm{CC}=\frac{1}{I J K}\left(\sum_{k=1}^{K} \rho\left(\mathcal{Y}_{:,:, k}, \widehat{\mathcal{Y}}_{:,:, k}\right)\right),
$$


where $\rho(\cdot, \cdot)$ is the Pearson correlation coefficient between the estimated and original spectral slices;

$$
\mathrm{SAM}=\frac{180}{\pi} \frac{1}{I J} \sum_{n=1}^{I J} \arccos \left(\frac{\boldsymbol{Y}_{n,:}^{(3)} \widehat{\boldsymbol{Y}}_{n,:}^{(3)}}{\left\|\boldsymbol{Y}_{n,:}^{(3)}\right\|_{2}\left\|\widehat{\boldsymbol{Y}}_{n,:}^{(3)}\right\|_{2}}\right),
$$

which computes the angle between original and estimated fibers;

$$
\text { ERGAS }=\frac{100}{d} \sqrt{\frac{1}{I J K} \sum_{k=1}^{K} \frac{\left\|\widehat{\mathcal{Y}}_{:,:, k}-\mathcal{Y}_{:,, i}\right\|_{F}^{2}}{\mu_{k}^{2}}},
$$

where $\mu_{k}^{2}$ is the mean value of $\hat{\mathcal{Y}}_{:,, k}$. ERGAS represents the relative dimensionless global error between the SRI and the estimate, which is the root mean-square error averaged by the size of the SRI. We also show the computational time for each algorithm, given by the tic and toc functions of MATLAB.

\section{Semi-real data: comparison with other methods}

In this subsection, we showcase the capabilities of SCOTT and B-SCOTT and compare them with state-of-the-art methods.

1) Non-blind algorithms: We compare the performance of non-blind algorithms (i.e STEREO and its initialization algorithm TenRec, and SCOTT). We test various ranks for both algorithms. For STEREO and TenRec, we use the implementation] of [9], available online at [30]. In other subsections, we use our implementation with fast solvers for the Sylvester equations (see Appendix A). For HySure [6], the groundtruth number of materials $E$ is chosen as the number of endmembers as in [9]. This algorithm is applied in a non-blind fashion, meaning that the spatial 8 and spectral degradation operators are not estimated but obtained from $\boldsymbol{P}_{1}, \boldsymbol{P}_{2}$ and $\boldsymbol{P}_{M}$. The same model is applied to the FUSE algorithm [31]. As a comparison, we also show the performance of B-SCOTT when no splitting is performed.

The first dataset we consider is Indian Pines, where $\mathcal{Y} \in$ $\mathbb{R}^{144 \times 144 \times 200}$ is degraded by a LANDSAT sensor for the MSI and a downsampling ratio $d=4$ for the HSI. The results are presented in Tables $\mathrm{I}$ and $\mathrm{II}$, and Figure 3 . In Table $\mathrm{I}$ and following, the numbers between brackets represent the multilinear rank used for the Tucker approach.

In the noiseless case (see Table \), we can see that for multilinear ranks chosen in the recoverability region (see Figure 11, SCOTT yields similar performance to the one of STEREO with lower computation time. Moreover, contrary to [9] (where $F=50$ is taken for STEREO), we found out that tensor rank $F=100$ yields better performance.

In Table II white Gaussian noise is added to $\mathcal{Y}_{H}$ and $\mathcal{Y}_{M}$ with an input SNR of $25 \mathrm{~dB}$. In this case, as in [9], tensor rank $F=50$ yields better performance. For $F=100$, TenRec gives slightly better performance than STEREO. Compared with the noiseless case, the performance of STEREO and TenRec deteriorate slightly, while we observe a bigger loss

\footnotetext{
${ }^{7}$ TenRec may be also made faster, e.g., by using algebraic algorithms for $\mathrm{CP}$ approximation [29], but we did not optimize its speed in this paper.

${ }^{8}$ In fact, HySure has a different, convolutional degradation model, that is not necessarily separable.
}

\begin{tabular}{c|c|c|c|c|c} 
Algorithm & R-SNR & CC & SAM & ERGAS & time \\
\hline STEREO 50 & 26.89 & 0.88 & 2.26 & 1.04 & 2.17 \\
STEREO 100 & 28.46 & 0.91 & 2.03 & 0.89 & 3.31 \\
SCOTT [40,40,6] & 26.28 & 0.88 & 2.36 & 1.08 & 0.29 \\
SCOTT [30,30,16] & 25.03 & 0.87 & 2.53 & 1.2 & 0.41 \\
SCOTT [70,70,6] & 26.34 & 0.88 & 2.52 & 1.13 & 0.35 \\
SCOTT [24,24,25] & 25.06 & 0.88 & 2.45 & 1.18 & 0.16 \\
B-SCOTT [40,40,6] & 25.12 & 0.87 & 2.76 & 1.25 & 0.11 \\
HySure E = 16 & 26.43 & 0.87 & 2.5 & 1.1 & 15.18 \\
FUSE & 21.95 & 0.81 & 3.91 & 1.91 & 0.33 \\
TenRec F = 50 & 26.82 & 0.88 & 2.27 & 1.05 & 0.84 \\
TenRec F = 100 & 28.34 & 0.9 & 2.05 & 0.9 & 2.1 \\
TABLE I & TABLE I \\
INDIAN PINES (NON-BLIND ALGORITHMS), NO NOISE
\end{tabular}

\begin{tabular}{c|c|c|c|c|c} 
Algorithm & R-SNR & CC & SAM & ERGAS & time \\
\hline STEREO F = 50 & 25.36 & 0.85 & 2.62 & 1.2 & 2.11 \\
STEREO F = 100 & 24.01 & 0.82 & 3.16 & 1.39 & 3.27 \\
SCOTT [40,40,6] & 23.67 & 0.83 & 3.14 & 1.46 & 0.33 \\
SCOTT [30,30,16] & 22.85 & 0.84 & 3.47 & 1.41 & 0.43 \\
SCOTT [70,70,6] & 17.99 & 0.77 & 5.92 & 2.88 & 0.36 \\
SCOTT [24,24,25] & 23.69 & 0.85 & 3.08 & 1.32 & 0.17 \\
B-SCOTT [40,40,6] & 20.26 & 0.81 & 4.65 & 2.2 & 0.11 \\
HySure E = 16 & 20.44 & 0.72 & 4.83 & 2.09 & 15.71 \\
FUSE & 12.21 & 0.62 & 11.17 & 5.66 & 0.35 \\
TenRec F = 50 & 25.24 & 0.85 & 2.65 & 1.23 & 0.83 \\
TenRec F = 100 & 24.29 & 0.82 & 3.01 & 1.37 & 2.09 \\
TABLE II & 3.01 & Th
\end{tabular}

of performance for other methods, including SCOTT and BSCOTT.
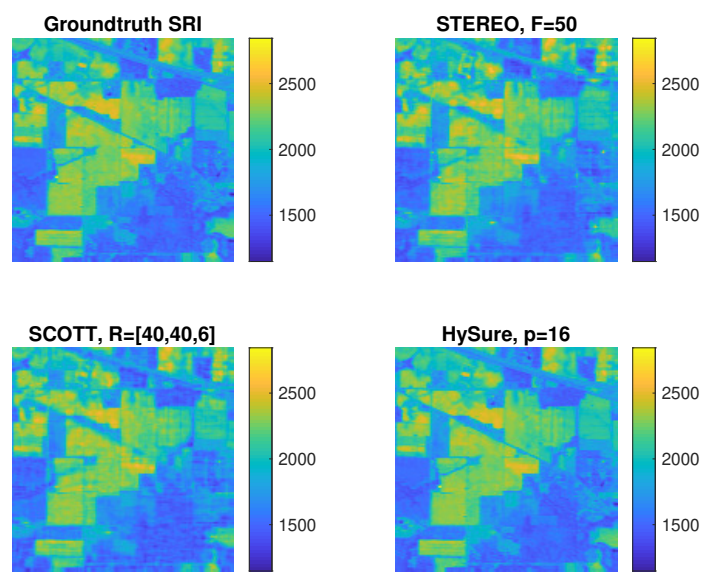

Fig. 3. Spectral slice 120 of the SRI, Indian Pines

The other dataset is the Salinas-A scene, where $\mathcal{Y} \in$ $\mathbb{R}^{80 \times 84 \times 204}$ is degraded with QuickBird specifications and $d=4$ for the HSI. The results are presented in Table III and Figure 4

In [9], CP-rank $F=100$ is used for STEREO. However, we found out that for STEREO, tensor rank $F=50$ yields better reconstruction. In Figure 4, we can see that STEREO and SCOTT can recover accurately the SRI.

2) Blind algorithms: We now consider the case where the spatial degradation matrices $\boldsymbol{P}_{1}, \boldsymbol{P}_{2}$ are unknown and compare the performance of B-SCOTT with Blind-STEREO [9], SCUBA [11] and HySure. We also compare the proposed 


\begin{tabular}{c|c|c|c|c|c} 
Algorithm & R-SNR & CC & SAM & ERGAS & time \\
\hline STEREO F = 50 & 33.4 & 0.97 & 0.91 & 3.35 & 0.92 \\
STEREO F = 100 & 32.91 & 0.94 & 0.58 & 5.41 & 0.9 \\
SCOTT [40,40,6] & 31.52 & 0.95 & 0.71 & 4.92 & 0.1 \\
SCOTT [50,50,6] & 32.31 & 0.95 & 0.59 & 4.89 & 0.13 \\
SCOTT [70,70,6] & 32.89 & 0.95 & 0.48 & 4.89 & 0.28 \\
B-SCOTT [40,40,6] & 31.3 & 0.95 & 0.74 & 4.96 & 0.03 \\
HySure E = 6 & 31.59 & 0.95 & 0.65 & 4.96 & 4.72 \\
FUSE & 20.61 & 0.88 & 1.91 & 5.89 & 0.12 \\
TenRec F = 50 & 33.24 & 0.97 & 0.89 & 2.97 & 0.6 \\
TenRec F = 100 & 29.15 & 0.92 & 0.63 & 9.79 & 0.84 \\
& \multicolumn{3}{|c}{ TABLE III }
\end{tabular}

SALINAS A-SCENE (NON-BLIND ALGORITHMS)
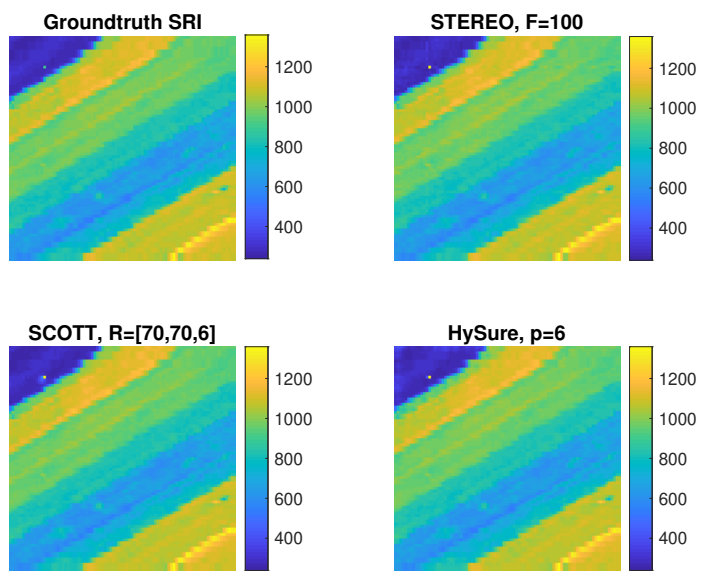

Fig. 4. Spectral slice 120 of the SRI, Salinas-A scene

blind algorithm to Blind-TenRec, the algebraic initialization of Blind-STEREO. White gaussian noise is added to the HSI and MSI, with a SNR of $15 \mathrm{~dB}$ and $25 \mathrm{~dB}$, respectively. We consider two other datasets; the first one is a portion of the Pavia University, where $\mathcal{Y} \in \mathbb{R}^{608 \times 366 \times 103}$ is degraded with QuickBird specifications for the MSI and $d=4$ for the HSI. We demonstrate the results in Table IV and Figure 5 for visual reconstruction. For B-SCOTT, in the case where $R=[152,84,3]$, no compression is performed. In the following tables, the numbers between parentheses denote the number of blocks in which the HSI and MSI are split. For SCUBA, the numbers between brackets represent $\left[F, R_{3}\right]$.

\begin{tabular}{c|c|c|c|c|c} 
Algorithm & R-SNR & CC & SAM & ERGAS & time \\
\hline SCUBA (4,4) [120,3] & 25.67 & 0.99 & 3.24 & 1.97 & 18.97 \\
B-SCOTT (4,4) [60,60,3] & 23.41 & 0.99 & 3.83 & 2.37 & 0.44 \\
B-SCOTT (4,4) [152,84,3] & 26.42 & 0.99 & 2.97 & 1.83 & 0.57 \\
B-SCOTT (4,4) [120,60,4] & 25.63 & 0.99 & 3.01 & 1.81 & 0.46 \\
SCUBA (8,8) [120,3] & 26.49 & 0.99 & 2.93 & 1.81 & 50.15 \\
B-SCOTT (8,8) [70,40,3] & 26.52 & 0.99 & 2.92 & 1.81 & 0.57 \\
B-STEREO F = 300 & 23.11 & 0.98 & 3.97 & 2.46 & 82.86 \\
HySure E = 9 & 26.27 & 0.99 & 2.83 & 1.74 & 115.39 \\
B-TenRec F = 300 & 22.52 & 0.98 & 4.06 & 2.65 & 29.51
\end{tabular}

PAVIA UNIVERSITY (BLIND ALGORITHMS)

In the second case, we consider the Cuprite dataset, where $\mathcal{Y} \in \mathbb{R}^{512 \times 614 \times 224}$ is degraded with LANDSAT specifications and $d=4$. The results are presented in Table $\mathrm{V}$ and Figure 6 .

These two previous examples show that, for different splittings, and ranks taken from [11], B-SCOTT yields the best
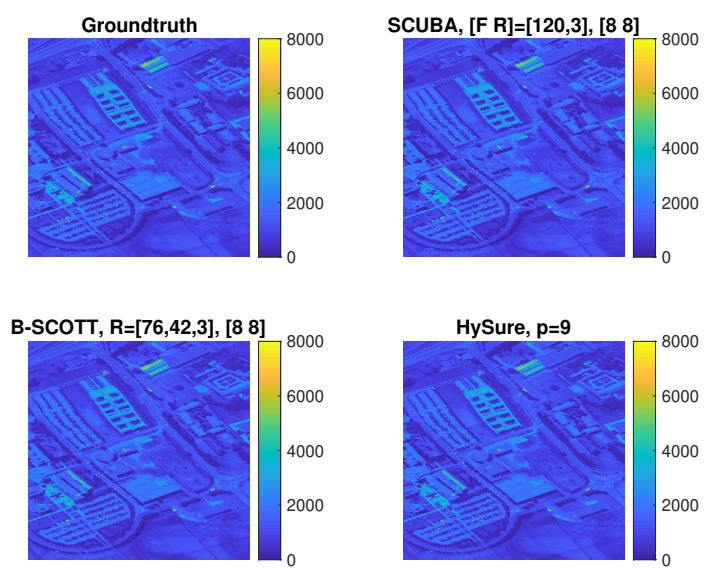

Fig. 5. Spectral slice 44 of the SRI, Pavia University

\begin{tabular}{c|c|c|c|c|c} 
Algorithm & R-SNR & CC & SAM & ERGAS & time \\
\hline SCUBA $(4,4)[45,3]$ & 31.71 & 0.97 & 1.12 & 6.57 & 12.58 \\
B-SCOTT $(4,4)[45,45,3]$ & 31.91 & 0.97 & 1.08 & 6.57 & 0.89 \\
B-SCOTT $(4,4)[60,60,3]$ & 33.02 & 0.98 & 1.03 & 6.58 & 1.19 \\
SCUBA $(8,8)[45,3]$ & 34.66 & 0.99 & 0.92 & 6.17 & 33.79 \\
B-SCOTT $(8,8)[45,45,3]$ & 34.7 & 0.99 & 0.91 & 6.19 & 1.25 \\
B-STEREO F = 150 & 29.87 & 0.97 & 1.37 & 7.35 & 56.42 \\
HySure E = 10 & 34.62 & 0.99 & 0.94 & 6.83 & 201.4 \\
B-TenRec F = 150 & 30.7 & 0.97 & 1.21 & 6.48 & 12.67 \\
\multicolumn{5}{c}{ TABLE V } \\
CUPRITE (BLIND ALGORITHMS)
\end{tabular}

performance. For certain multilinear ranks, it even outperforms SCUBA with lower computation time. Moreover, it outperforms Blind-STEREO and Blind-TenRec. In terms of visual reconstruction, our algorithm can recover accurately the details of the groundtruth SRI, even though the spatial degradation matrices are unknown.
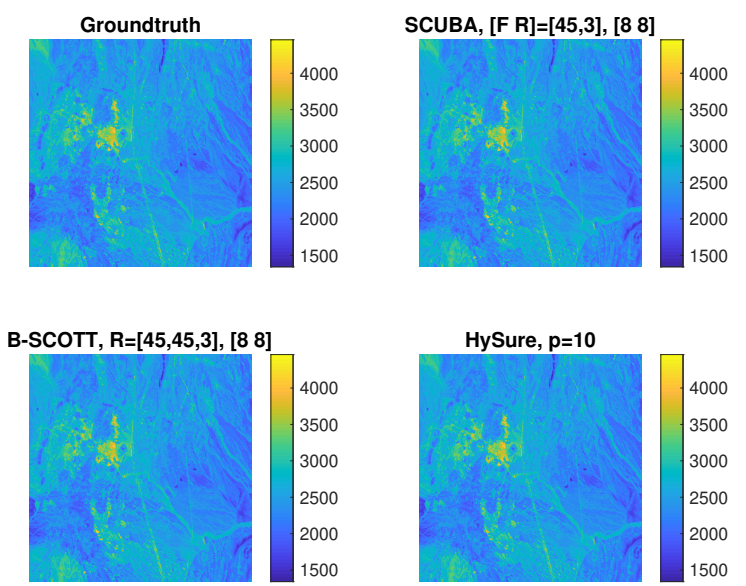

Fig. 6. Spectral slice 44 of the SRI, Cuprite

3) Hyperspectral pansharpening: Next, we address the pansharpening problem, which consists in fusion of a hyperspectral image and a panchromatic image (PAN) $\mathcal{Y}_{P} \in$ $\mathbb{R}^{I \times J \times 1}$. In this case, the spectral degradation matrix is obtained by averaging over the full spectral range of the groundtruth SRI, so that $\boldsymbol{P}_{\boldsymbol{M}} \in \mathbb{R}^{1 \times K}$. CP-based algorithms are not applicable, since their initialization is based on the 
CPD of the MSI (which is a matrix in the case of PAN images). In Table VI the metrics are shown for different multilinear ranks for the Indian Pines dataset. We also compare our results to those of HySure. We can see that even though the only possible value of $R_{3}$ is 1 for B-SCOTT, the algorithm still manages to yield a good recovery of the SRI. On the other hand, SCOTT can also recover the SRI accurately, but is more sensitive to the choice of the multilinear rank.

\begin{tabular}{c|c|c|c|c|c} 
Algorithm & R-SNR & CC & SAM & ERGAS & time \\
\hline SCOTT [24,24,25] & 20.47 & 0.77 & 4.41 & 1.95 & 0.22 \\
SCOTT [30,30,16] & 18.04 & 0.69 & 5.68 & 2.59 & 0.43 \\
SCOTT [35,35,6] & 14.61 & 0.54 & 7.84 & 3.89 & 0.89 \\
HySure E = 16 & 20.67 & 0.76 & 4.24 & 1.99 & 14.39 \\
BSCOTT (4,4) [24,24,1] & 19.78 & 0.72 & 5.07 & 2.19 & 0.28 \\
BSCOTT (4,4) [35,35,1] & 19.79 & 0.72 & 5.07 & 2.19 & 0.12 \\
TABLE VI \\
INDIAN PINES (PANSHARPENING)
\end{tabular}

\section{Synthetic examples}

In most cases, generic recoverability conditions proposed in [9] are less restrictive than that of the Tucker approach. The tensor rank $F$ can be larger than the dimensions of the SRI, while the multilinear ranks are bounded by its dimensions. This gives the CP-based model better modelling power than the Tucker-based model, as shown for real data: regardless of the computation time, STEREO gives better performance than SCOTT. However, there may exist deterministic cases in which the Tucker recoverability conditions are satisfied while nothing can be concluded from the results of [9]. The goal of this subsection is to provide synthetic examples for such situations in the noiseless and noisy cases. While these examples do not necesserily look like realistic hyperspectral images, they do help to better understand the recoverability conditions of the SRI and to evaluate their impact on the estimation performance.

1) Generating synthetic SRI: First, we explain how the synthetic SRI $\mathcal{Y} \in \mathbb{R}^{I \times J \times K}$ are generated. We consider $N$ spectral signatures $s_{1}, \ldots, s_{N}$ obtained from the Indian Pines groundtruth data [28]. The SRI is split into $M^{2}$ equal blocks along the spatial dimensions. In each $\frac{I}{M} \times \frac{J}{M}$ block, at most one material is active, indicated by a number in the corresponding cell of a parcel map (see Table [VII for an example).

Formally, the SRI is computed as

$$
\begin{aligned}
& \mathcal{Y}=\sum_{n=1}^{N} \boldsymbol{A}_{n} \otimes \boldsymbol{s}_{n}, \\
& \begin{array}{|l|l|}
\hline 1 & 2 \\
\hline 2 & \\
\hline
\end{array} \\
& \text { TABLE VII } \\
& \text { PARCEL MAP FOR } N=2
\end{aligned}
$$

where the abundance map $\boldsymbol{A}_{n}$ is a block matrix with Gaussians of fixed size present on the blocks corresponding to material $n$ in the parcel map.

For instance, we consider the case presented in Table VII, the two abundance maps are

$$
\boldsymbol{A}_{1}=\left[\begin{array}{cc}
\boldsymbol{H} & 0 \\
0 & 0
\end{array}\right], \quad \boldsymbol{A}_{2}=\left[\begin{array}{cc}
0 & \boldsymbol{H} \\
\boldsymbol{H} & 0
\end{array}\right],
$$

where $\boldsymbol{H}$ is a $60 \times 60$ Gaussian with $\sigma=20$. To illustrate this example, we show in Figure 7 two spectral bands of $\mathcal{Y}$.
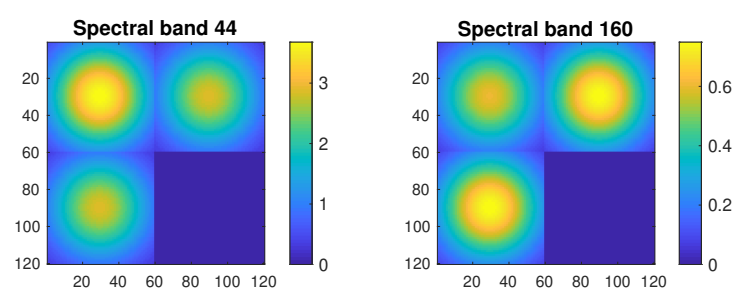

Fig. 7. Spectral bands of the synthetic SRI with $N=2$

2) Non-existing low-rank approximations: Let us consider the example introduced in Table VII Due to separability of the Gaussians, $\mathcal{Y}$ has the following multilinear decomposition:

$$
\begin{array}{r}
\mathcal{Y}=\llbracket \mathcal{G} ; \boldsymbol{U}, \boldsymbol{V}, \boldsymbol{S} \rrbracket \\
\text { where } \mathcal{G}_{:,:, 1}=\left[\begin{array}{ll}
1 & 0 \\
0 & 0
\end{array}\right], \mathcal{G}_{:, 2,2}=\left[\begin{array}{ll}
0 & 1 \\
1 & 0
\end{array}\right] \\
\boldsymbol{U}=\boldsymbol{V}=\left[\begin{array}{cc}
H & 0 \\
0 & H
\end{array}\right] \text { and } \boldsymbol{S}=\left[\begin{array}{ll}
\boldsymbol{s}_{1} & \boldsymbol{s}_{2}
\end{array}\right]
\end{array}
$$

The multilinear rank of $\mathcal{Y}$ is equal to $(2,2,2)$, while the tensor rank of $\mathcal{Y}$ is equal to the tensor rank of $\mathcal{G}$, which is known to be equal to $F=3$ [32, Ex. 2], [33, Ex. 6.6]. This is a wellknown case where the best rank-2 $\mathrm{CP}$ approximation does not exist [34], [35], thus we can expect problems with the CPbased approach.

We generate $\mathcal{Y}_{H}$ and $\mathcal{Y}_{M}$ with a downsampling ratio of $d=4$ for the HSI and LANDSAT specifications for the MSI (see Appendix B. No noise is added to the MSI and HSI. We run STEREO and TenRec for $F$ in $[1: 40]$ and SCOTT for $R_{1}=R_{2}$ in [1:40] and $R_{3}$ in [1:10] under recoverability conditions. For each algorithm, we compute the R-SNR as a function of the rank; the results are provided on Figure 8 . As a comparison, on the same plot as STEREO and TenRec, we plot the results of SCOTT for $R_{3}=N$ and $R_{1}=R_{2}=F$.
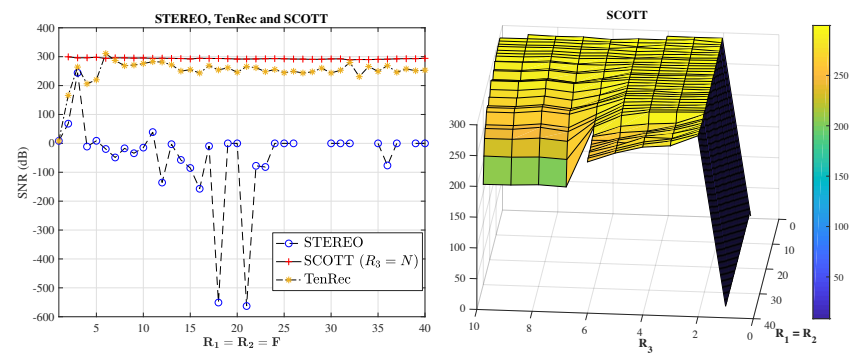

Fig. 8. R-SNR as a function of the rank

For SCOTT, the best reconstruction error (given by R-SNR) is obtained for $R_{3}=N$ and is rather insensitive to the choice of $R_{1}=R_{2}$. $R_{3}$ can also be chosen larger than $N$ without significant loss of performance. For STEREO, only rank $F=$ 3 allows for an accurate reconstruction of the SRI. For other tensor ranks, either the algorithm breaks (when no point is plotted, e.g. $F=32$ ) or leads to inaccurate recovery. TenRec however achieves the correct recovery for a wide range of tensor ranks. We can see that in this case, performing STEREO iterations after TenRec leads to a loss of performance. We 
believe that this is due to the presence of colinear factors in the approximation causing ill-conditioning of ALS iterations. However, for noisy or real examples, this phenomenon is not likely to occur.

3) Higher rank and noisy example: We also consider a slightly more realistic scenario. The following example is made of $N=7$ materials, generated similarly to the previous example, as illustrated in Table VIII and Figure 9 The abundance maps are arranged along an anti-diagonal pattern, in a similar fashion as for the Salinas-A dataset.

\begin{tabular}{|l|l|l|l|}
\hline 1 & 2 & 3 & 4 \\
\hline 2 & 3 & 4 & 5 \\
\hline 3 & 4 & 5 & 6 \\
\hline 4 & 5 & 6 & 7 \\
\hline \multicolumn{5}{|c|}{}
\end{tabular}

PARCEL MAP FOR $N=7$
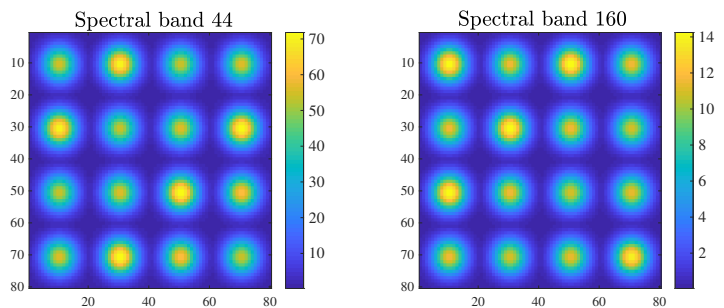

Fig. 9. Spectral bands of the synthetic SRI with $N=7$

In this example, $\mathcal{Y} \in \mathbb{R}^{80 \times 80 \times 200}$ is degraded with QuickBird specifications for the MSI and $d=4$ for the HSI. White Gaussian noise is added to the degraded images with an input SNR of $35 \mathrm{~dB}$. The multilinear rank of the SRI is $\boldsymbol{R}=(4,4,7)$ while we do not know the tensor rank. Similarly, we run both algorithms with the same setup as in the previous example, including a comparison of STEREO and TenRec, and SCOTT for $R_{3}=N$ and an overestimated $R_{3}=15$. Results are presented in Figure 10

For SCOTT, the best R-SNR is obtained for $\boldsymbol{R}=(4,4,7)$, which is the multilinear rank of the noiseless tensor. Moreover, the best reconstruction error is obtained for $R_{3}=N$ : in this case, the performance of SCOTT is better than that of CPbased approaches. SCOTT is also robust to an overestimation of $R_{3}$ or $R_{1}=R_{2}$. TenRec and STEREO have almost the same performance, which is lower than that of SCOTT in this example.
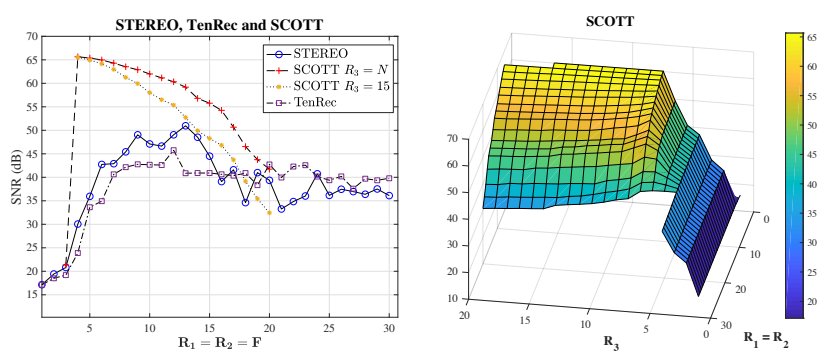

Fig. 10. R-SNR as a function of the rank
4) Block tensor: Here, we provide an example in which the CPD of the MSI is not unique but the CP approach still achieves the correct recovery of the SRI. This dataset is made of $N=6$ materials with spatial degradation ratio of $d=4$ for the HSI and Quickbird specifications for the MSI so that $K_{M}<N$. Each abundance map is made of two $10 \times 10$ Gaussians of width $\sigma=4$, as in Table IX.

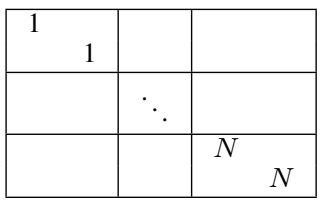

TABLE IX

PARCEL MAP FOR BLOCKED TENSOR, $N=6$

In this example, the tensor rank of $\mathcal{Y}$ is $F=12$ while the multilinear rank is $\boldsymbol{R}=(12,12,6)$. The $\mathrm{CP}$ decompositions of both MSI and HSI are not unique, but the recoverability conditions given in Corollary V.3 are satisfied. This is an example of a tensor admitting a block-term decomposition, where the abundance maps in 20 corresponding to different materials are not rank-one. While this is not a realistic example due to small ranks of abundance maps, it is inspired by the standard linear mixing model [1] with few materials.
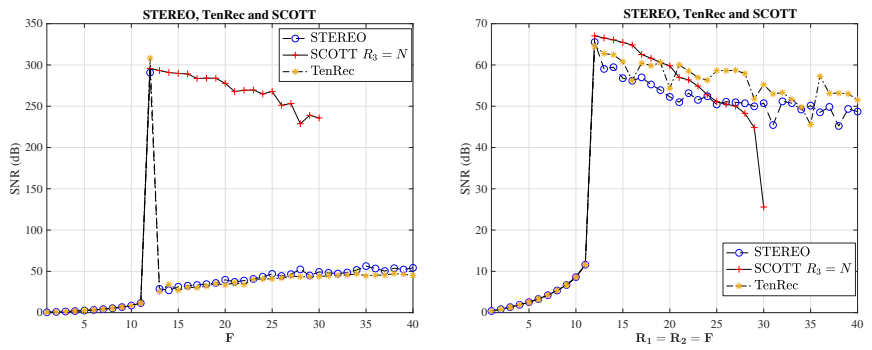

Fig. 11. R-SNR as a function of the rank in the noiseless case (left) and with $35 \mathrm{~dB}$ input SNR (right)

In Figure 11, we show the R-SNR as a function of the rank for STEREO and SCOTT for the noiseless and noisy cases. In the noiseless case, under recoverability conditions, our Tuckerbased approach provides good reconstruction for a variety of ranks and $R_{3} \geq N$. For STEREO and TenRec, we can see that even though the CP model is not identifiable, $F=12$ allows correct reconstruction of the SRI with almost the same performance as that of SCOTT for $\boldsymbol{R}=(12,12,6)$ (up to machine precision).

This example corroborates Corollary V.3 and shows that identifiability of the CP model (as it is formulated in [9]) is not necessary to reconstruct $\mathcal{Y}$ accurately, and partial uniqueness may be sufficient.

In the noisy case, the three algorithms have almost the same performance for $R_{1}=R_{2}=F=12$. However, for $F \geq 21$, TenRec gives better performance than SCOTT, and for $F \geq$ 26, STEREO overcomes our approach.

\section{E. Choice of multilinear ranks in the presence of noise}

In Section V, we provided a theorem for recoverability of the SRI. In this subsection, we show that the conditions of 
Theorem V.1 also give hints on choosing the multilinear ranks for HSR in "signal+noise" and semireal scenarios.

1) Singular values of the unfoldings: Motivated by step 1 of Algorithm 5, where the factor matrices $\boldsymbol{U}, \boldsymbol{V}, \boldsymbol{W}$ are computed by HOSVD of the HSI and MSI, and by the first set of conditions in Theorem V.1. we look at the singular values of $\boldsymbol{Y}_{M}^{(1)}, \boldsymbol{Y}_{M}^{(2)}$ and $\boldsymbol{Y}_{H}^{(3)}$.

We first consider the synthetic data from Figure 7 with $N=$ 2 materials, and add white Gaussian noise to $\mathcal{Y}_{H}$ and $\mathcal{Y}_{M}$ with different SNR: 20dB, 35dB, 60dB and no noise. In Figure 12 . we plot the 15 first singular values of the unfoldings on a semi-log scale.

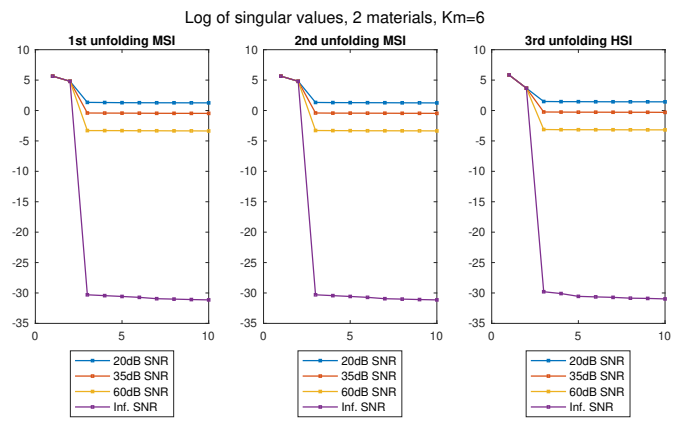

Fig. 12. Logarithm of the first 15 singular values for the three unfoldings

We can see that for all the considered noise levels, the singular values are well separable. The corners of the curves at singular values $(2,2,2)$ are coherent with the theoretical multilinear rank of the synthetic SRI.

We now consider the semi-real datasets Indian Pines and Salinas-A and plot the singular values of the unfoldings on a semi-log scale on Figures 13 and 14
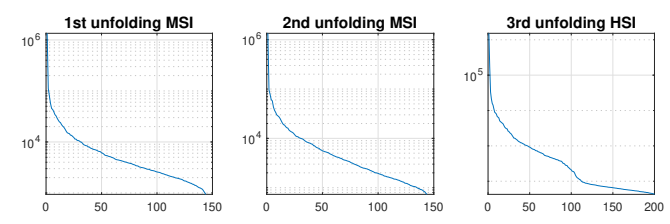

Fig. 13. Singular values for the three unfoldings, Indian Pines
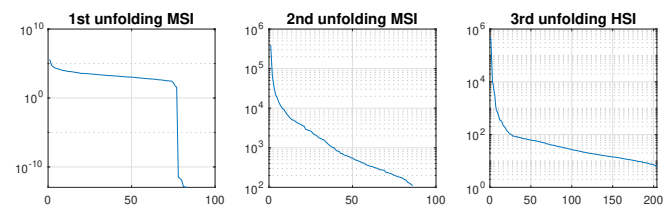

Fig. 14. Singular values for the three unfoldings, Salinas-A scene

In the semi-real cases, a clear corner in the singular value curves cannot be found, because these examples do not correspond to a "low-rank signal+noise" scenario, contrary to the case of synthetic data. Moreover, the HSI and MSI are not necessarily low-rank: hence, the Tucker approach only performs a low-rank approximation of the data. Hence, the SVD of the unfolding does not provide as much information as for the synthetic case, in which the groundtruth data are explicitly designed to be low-rank.

2) Influence on the reconstruction error: Next, we consider the R-SNR and cost function $f_{T}$ as functions of the multilinear rank. We run SCOTT for the ranks $R_{1}=R_{2}$ in $[10: 50]$ and $R_{3}$ in $[2: 25]$ for which the recoverability condition holds (see Section $\sqrt{\mathrm{V}}$ ), and two semi-real datasets: Indian Pines and Salinas-A scene. The results are shown in Figures 15 and 16 . respectively.
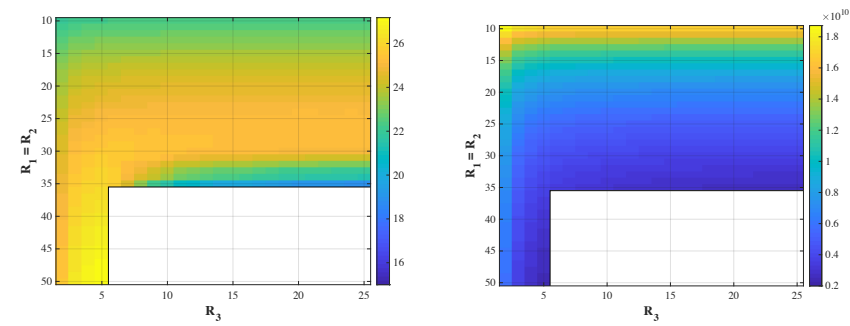

Fig. 15. R-SNR (left) and $f_{T}$ (right) as functions of $R_{1}$ and $R_{3}$, Indian Pines
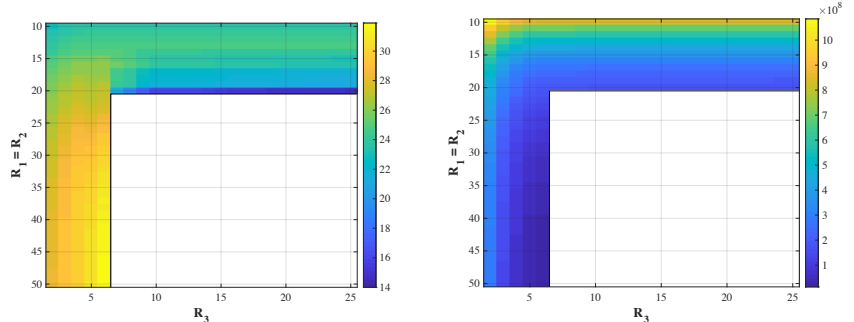

Fig. 16. R-SNR (left) and $f_{T}$ (right) as functions of $R_{1}$ and $R_{3}$, Salinas-A

While the cost function decreases as $R_{1}$ and $R_{3}$ increase, the best reconstruction error (given by R-SNR) is achieved in one of the two recoverability subregions in Fig. 1) $(a)\left(R_{3} \geq\right.$ $K_{M}$ and $\left.R_{1} \leq I_{H}\right)$ and $(b)\left(R_{3} \leq K_{M}\right.$ and $\left.R_{1} \geq I_{H}\right)$. For subregion $(b)$, the best performance is achieved when $R_{3}=$ $K_{M}$ and $R_{1}$ as large as possible, while for subregion (a), we notice a sharp drop of the R-SNR near $R_{1}=I_{H}$.

The drop of the performance in subregion (a) can be explained by looking at the condition number of the matrix $\boldsymbol{X}^{\top} \boldsymbol{X}$ that is used to compute the core tensor $\widehat{\mathcal{G}}$. For the subregion (a), due to properties of Kronecker products [22, Theorems 13.12 and 13.16], we have that

$$
\begin{aligned}
& \operatorname{cond}\left\{\boldsymbol{X}^{\top} \boldsymbol{X}\right\}:=\frac{\sigma_{\max }\left(\boldsymbol{X}^{\top} \boldsymbol{X}\right)}{\sigma_{\min }\left(\boldsymbol{X}^{\top} \boldsymbol{X}\right)} \\
& =\frac{\lambda \sigma_{\max }^{2}\left(\boldsymbol{P}_{M} \widehat{\boldsymbol{W}}\right)+\sigma_{\max }^{2}\left(\boldsymbol{P}_{1} \widehat{\boldsymbol{U}}\right) \sigma_{\max }^{2}\left(\boldsymbol{P}_{2} \widehat{\boldsymbol{V}}\right)}{\sigma_{\min }^{2}\left(\boldsymbol{P}_{1} \widehat{\boldsymbol{U}}\right) \sigma_{\min }^{2}\left(\boldsymbol{P}_{2} \widehat{\boldsymbol{V}}\right)} .
\end{aligned}
$$

Note that $\sigma_{\max }\left(\boldsymbol{P}_{M} \widehat{\boldsymbol{W}}\right)$ does not decrease when we increase $R_{3}$ and $R_{3} \leq K_{M}$. Hence we can get a lower bound on $\operatorname{cond}\left\{\boldsymbol{X}^{\top} \boldsymbol{X}\right\}$ by setting $R_{3}=K_{M}$.

In Figure 17, for the Indian Pines dataset we plot on a semi-log scale the lower bound $\operatorname{cond}\left\{\boldsymbol{X}^{\top} \boldsymbol{X}\right\}$ as functions of $R_{1}=R_{2}$, for $R_{3}=6$ as well as $\sigma_{\max }\left(\boldsymbol{P}_{M} \widehat{\boldsymbol{W}}\right)$; since the latter almost does not change, the lower bound is tight. In Figure 17, we see that there is a highest relative increase of the condition number around $R_{1}=R_{2}=32$, which coincides 

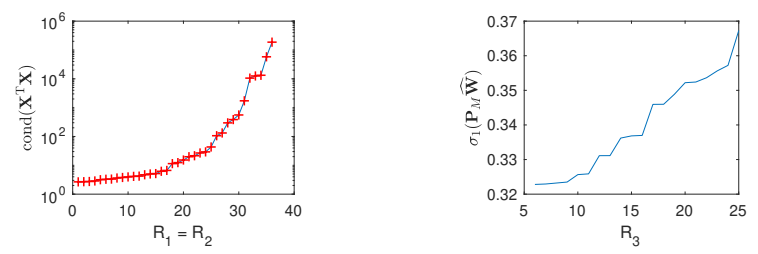

Fig. 17. Left: $\log \left(\operatorname{cond}\left\{\boldsymbol{X}^{\top} \boldsymbol{X}\right\}\right)$ depending on $R_{1}=R_{2}$ for $R_{3}=K_{M}$; right: $\sigma_{1}\left(\boldsymbol{P}_{M} \widehat{\boldsymbol{W}}\right)$, Indian Pines

with the point of the performance drop in Figure 15 Similar behaviour can be observed for the Salinas dataset on Figure 18 .
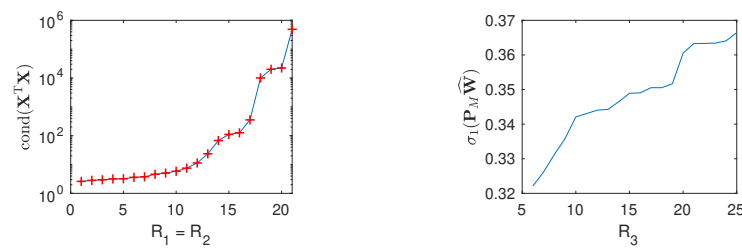

Fig. 18. Left: $\log \left(\operatorname{cond}\left\{\boldsymbol{X}^{\top} \boldsymbol{X}\right\}\right)$ depending on $R_{1}=R_{2}$ for $R_{3}=K_{M}$; right: $\sigma_{1}\left(\boldsymbol{P}_{M} \widehat{\boldsymbol{W}}\right)$, Salinas-A scene

All in all, based on the above examples, we can conclude that if we are in subregion (b), the $R_{3}$ should be taken as large as possible $\left(R_{3}=K_{M}\right)$, while in the subregion (a) $R_{1}$, $R_{2}$ should be taken as large as possible while maintaining the condition number to a reasonable value.

\section{F. Recovery of spectral signatures}

Since correct recovery of spectral signatures is quite important for further processing of hyperspectral images, we would like see whether SCOTT is able to do that. We consider the Indian Pines dataset, where groundtruth data (see Fig. 19) is available, splitting the image into 16 regions. We will consider three representative ranks: $[40,40,6],[30,30,16]$, and $[24,24,25]$, and compare them to STEREO $(F=100)$.

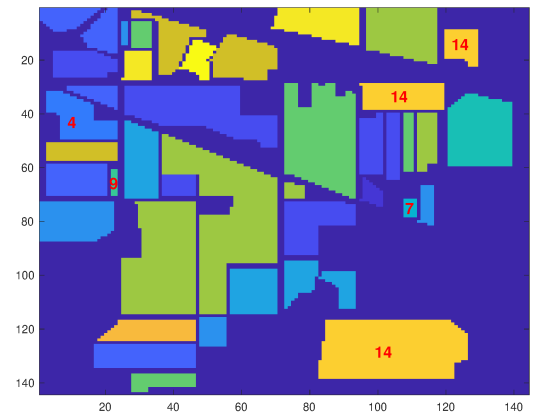

Fig. 19. Groundtruth image for Indian Pines dataset. Materials 4,7,9,14 are marked in red.

We do not perform a proper hyperspectral unmixing, and compute the spectral signatures by averaging across the regions. We selected four representative signatures corresponding to materials 4,7,9 and 14, which are plotted in Figure VI-F. Note that materials 7 and 9 are scarce in the groundtruth SRI (resp. 28 and 20 pixels), whereas materials 4 and 14 are more abundant (resp. 237 and 1265 pixels).

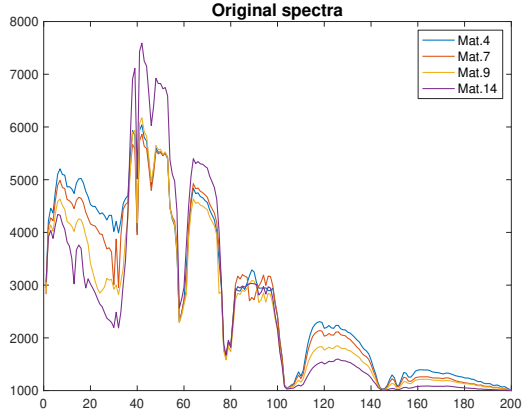

Fig. 20. Original spectral signature for materials $4,7,9$ and 14

In Figure 21 we plot relative errors of the reconstruction of spectra by different methods. As expected, for materials 7 and 9, the discrepancy between the original spectra and the spectra obtained from estimated SRI is bigger than for materials 4 and 14. This can be explained by the scarcity of sources 7 and 9 compared to sources 4 and 14 .
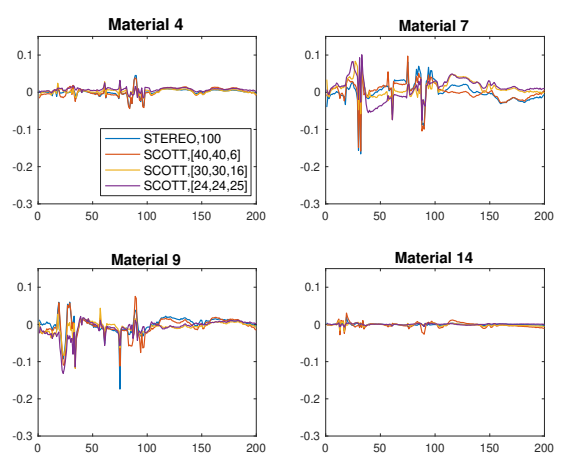

Fig. 21. Residual errors for the three considered ranks and four materials

In Figure 22, we have a closer look at the spectra at spectral bins 80 to 100 . We can see that for abundant materials (4 and 14), all the algorithms estimate well the spectra. For the scarce materials it is important to choose the rank large enough, in particular $R_{3}=16$ and $R_{3}=25$ yield better reconstruction than $R_{3}=6$, and also than STEREO, even with $F=100$.
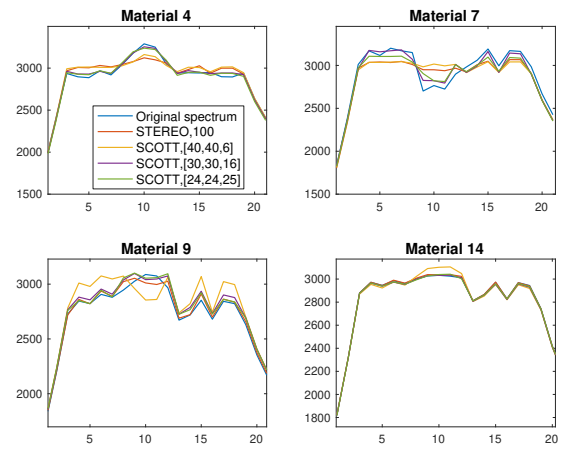

Fig. 22. Materials at spectral bins 80 to 100. Groundtruth (black), SCOTT $(40,40,6)$ (red), SCOTT $[30,30,16]$ (yellow), SCOTT $(24,24,25)$ (purple), STEREO $F=100$ (green). 


\section{CONCLUSION}

In this paper, we proposed a novel coupled Tucker model for hyperspectral superresolution. We showed that the model is recoverable, that is, it allows for an unique recovery of the SRI for a wide range of multilinear ranks. We proposed two very simple SVD-based algorithms that can be used for the super-resolution problem, for known and unknown degradation operators, and for the case of pansharpening. The algorithms are very fast, but produce the results that are comparable with the CP-based approaches. This work opens new perspectives on using various tensor factorizations for hyperspectral super-resolution. Still several interesting questions remain, for example, how to enlarge the recoverability range for the multilinear rank. Estimating the multilinear rank of the Tucker decomposition still remains an open problem; the question of optimal splitting the data into non-overlapping subtensors in B-SCOTT also needs to be further investigated.

\section{APPENDIX A}

\section{SOLVING NORMAL EQUATIONS AS GENERALIZED SYLVESTER EQUATIONS}

Equation (7) can be seen as a generalized Sylvester equation of the form

$$
\boldsymbol{A} \widehat{G} B+C \widehat{G} D=E,
$$

where $\boldsymbol{G}$ is an unfolding of $\widehat{\mathcal{G}}$.

We propose two options for converting (7) into (21). In the first case, $\widehat{\boldsymbol{G}}=\mathcal{G}^{(3)} \in \mathbb{R}^{R_{1} R_{2} \times R_{3}}$,

$$
\begin{aligned}
& \boldsymbol{A}=\left(\boldsymbol{U}^{\top} \boldsymbol{P}_{1}^{\top} \boldsymbol{P}_{1} \boldsymbol{U}\right) \otimes\left(\boldsymbol{V}^{\top} \boldsymbol{P}_{2}^{\top} \boldsymbol{P}_{2} \boldsymbol{V}\right), \quad \boldsymbol{B}=\boldsymbol{I}_{R_{3}}, \\
& \boldsymbol{C}=\boldsymbol{I}_{R_{1} R_{2}}, \quad \boldsymbol{D}=\lambda\left(\boldsymbol{W}^{\top} \boldsymbol{P}_{M}^{\top} \boldsymbol{P}_{M} \boldsymbol{W}\right),
\end{aligned}
$$

and $\boldsymbol{E} \in \mathbb{R}^{R_{1} R_{2} \times R_{3}}$ is a matricization of $\boldsymbol{X}^{\top} \boldsymbol{z}$.

In the second case, $\widehat{\boldsymbol{G}}=\mathcal{G}^{(1)^{\top}} \in \mathbb{R}^{R_{1} \times R_{2} R_{3}}$,

$$
\begin{aligned}
& \boldsymbol{A}=\boldsymbol{U}^{\top} \boldsymbol{P}_{1}^{\top} \boldsymbol{P}_{1} \boldsymbol{U}, \quad \boldsymbol{B}=\boldsymbol{I}_{R_{3}} \otimes\left(\boldsymbol{V}^{\top} \boldsymbol{P}_{2}^{\top} \boldsymbol{P}_{2} \boldsymbol{V}\right), \\
& \boldsymbol{C}=\boldsymbol{I}_{R_{1}}, \quad \boldsymbol{D}=\lambda\left(\boldsymbol{W}^{\top} \boldsymbol{P}_{M}^{\top} \boldsymbol{P}_{M} \boldsymbol{W}\right) \otimes \boldsymbol{I}_{R_{2}},
\end{aligned}
$$

and $\boldsymbol{E} \in \mathbb{R}^{R_{1} \times R_{2} R_{3}}$ is a matricization of $\boldsymbol{X}^{\top} \boldsymbol{z}$.

The two options are equivalent and the fastest one is chosen according to the multilinear rank. As a rule of thumb, we decide to choose the first option in subregion $(a)$ of Figure 1 and the second option in subregion $(b)$. The complexity for solving the generalized Sylvester equation 21 is thus $O\left(\mathrm{~m}^{3}+\right.$ $\left.n^{3}\right)$ flops for $\widehat{\boldsymbol{G}} \in \mathbb{R}^{m \times n}$ if fast solvers, such as HessenbergSchur or Bartels-Stewart methods [18], [19], [36], are used.

\section{APPENDIX B}

\section{DEGRADATION MATRICES}

Here, we explain in details how the degradation matrices are constructed. For this appendix, we consider that $\boldsymbol{P}_{1}=\boldsymbol{P}_{2}$. As in [9], $\boldsymbol{P}_{1}$ is constructed as $\boldsymbol{P}_{1}=\boldsymbol{S}_{1} \boldsymbol{T}_{1}$, where $\boldsymbol{T}_{1}$ is a blurring matrix and $\boldsymbol{S}_{1}$ is a downsampling matrix.

The blurring matrix is constructed from a Gaussian blurring kernel $\phi \in \mathbb{R}^{q \times 1}$ (in our case, $q=9$ ) with a standard deviation $\sigma$. For $m=1, \ldots, q$ and $m^{\prime}=m-\left\lceil\frac{q}{2}\right\rceil$, we have

$$
\phi(m)=\frac{1}{\sqrt{2 \pi \sigma^{2}}} \exp \left(\frac{-m^{\prime 2}}{2 \sigma^{2}}\right) .
$$

Thus, $\boldsymbol{T}_{1} \in \mathbb{R}^{I \times I}$ can be seen as

$$
\boldsymbol{T}_{1}=\left[\begin{array}{cccccc}
\phi\left(\left\lceil\frac{q}{2}\right\rceil\right) & \ldots & \phi(q) & 0 & \ldots & 0 \\
\vdots & \ddots & & \ddots & \ddots & \vdots \\
\phi(1) & & \ddots & & \ddots & 0 \\
0 & \ddots & & \ddots & & \phi(q) \\
\vdots & \ddots & \ddots & & \ddots & \vdots \\
0 & \ldots & 0 & \phi(1) & \ldots & \phi\left(\left\lceil\frac{q}{2}\right\rceil\right)
\end{array}\right] .
$$

The downsampling matrix $\boldsymbol{S}_{1} \in \mathbb{R}^{I_{H} \times I}$, with downsampling ratio $d$, is made of $I_{H}$ independant rows such that for $i=1, \ldots, I_{H},\left(\boldsymbol{S}_{1}\right)_{i, 2+(i-1) d}=1$ and the other coefficients are zeros.

The spectral degradation matrix $\boldsymbol{P}_{M} \in \mathbb{R}^{K_{M} \times K}$ is a selection-averaging matrix, Each row represents a spectral range in the MSI; coefficients are set to ones for common bands with the SRI, and zeros elsewhere. The coefficients are averaged per-row. Below, we give an example of a $2 \times 6$ matrix:

$$
\left[\begin{array}{cccccc}
0 & \frac{1}{3} & \frac{1}{3} & \frac{1}{3} & 0 & 0 \\
0 & 0 & 0 & 0 & \frac{1}{2} & \frac{1}{2}
\end{array}\right] \text {. }
$$

\section{REFERENCES}

[1] N. Yokoya, C. Grohnfeldt, and J. Chanussot, "Hyperspectral and multispectral data fusion: A comparative review of the recent literature," IEEE Trans. Geosci. Remote Sens., vol. 5, no. 2, pp. 29-56, 2017.

[2] L. Loncan, L. B. de Almeida, J. M. Bioucas-Dias, X. Briottet, J. Chanussot, N. Dobigeon, S. Fabre, W. Liao, G. A. Licciardi, M. Simoes, J. Tourneret, M. A. Veganzones, G. Vivone, Q. Wei, and N. Yokoya, "Hyperspectral pansharpening: A review," IEEE Trans. Geosci. Remote Sens., vol. 3, no. 3, pp. 27-46, 2015.

[3] B. Aiazzi, L. Alparone, S. Baronti, A. Garzelli, M. Selva, and C. Chen, "25 years of pansharpening: a critical review and new developments," Signal and Image Process. for Remote Sens., pp. 533-548, 2011.

[4] N. Yokoya, T. Yairi, and A. Iwasaki, "Coupled nonnegative matrix factorization unmixing for hyperspectral and multispectral data fusion," IEEE Trans. Geosci. Remote Sens., vol. 50, no. 2, pp. 528-537, 2012.

[5] Q. Wei, J. Bioucas-Dias, N. Dobigeon, and J.-Y. Tourneret, "Hyperspectral and multispectral image fusion based on a sparse representation," IEEE Trans. Geosci. Remote Sens., vol. 53, no. 7, pp. 3658-3668, 2015.

[6] M. Simoẽs, J. Bioucas-Dias, L. B. Almeida, and J. Chanussot, "A convex formulation for hyperspectral image superresolution via subspace-based regularization," IEEE Trans. Geosci. Remote Sens., vol. 53, no. 6, pp. 33733388, 2015.

[7] Q. Wei, J. Bioucas-Dias, N. Dobigeon, and J.-Y. Tourneret, "Multiband image fusion based on spectral unmixing," IEEE Trans. Geosci. Remote Sens., vol. 54, no. 12, pp. 7236-7249, 2016.

[8] Q. Li, W.-K. Ma, and Q. Wu, "Hyperspectral super-resolution: Exact recovery in polynomial time," 2018 IEEE Statistical Signal Processing Workshop (SSP), pp. 378-382, 2018.

[9] C. I. Kanatsoulis, X. Fu, N. D. Sidiropoulos, and W.-K. Ma, "Hyperspectral super-resolution: A coupled tensor factorization approach," IEEE Trans. Signal Process., vol. 66, no. 24, pp. 6503-6517, 2018.

[10] J. Hatvani, A. Basarab, J.-Y. Tourneret, M. Gyöngy, and D. Kouamé, "A Tensor Factorization Method for 3D Super-Resolution with Application to Dental CT," IEEE Trans. Med. Imag., vol. 38, no. 6, pp. 1524-1531, 2019.

[11] C. I. Kanatsoulis, X. Fu, N. D. Sidiropoulos, and W. Ma, "Hyperspectral super-resolution: Combining low rank tensor and matrix structure," in 2018 25th IEEE International Conf. on Image Process. (ICIP), Oct 2018, pp. 3318-3322.

[12] L. De Lathauwer, B. De Moor, and J. Vandewalle, "A multilinear singular value decomposition," SIAM J. Matrix Anal. Appl., vol. 21, no. 4, pp. 1253-1278, 2000.

[13] X. He, L. Condat, J. M. Bioucas-Dias, J. Chanussot, and J. Xia, "A new pansharpening method based on spatial and spectral sparsity priors," IEEE Trans. on Image Process., vol. 23, no. 9, pp. 4160-4174, 2014. 
[14] C. Prévost, K. Usevich, P. Comon, and D. Brie, "Coupled tensor lowrank multilinear approximation for hyperspectral super-resolution," in 2019 IEEE Int. Conf. on Acoust., Speech and Sign. Process. (ICASSP), May 2019, pp. 5536-5540.

[15] P. Comon, "Tensors: A brief introduction," IEEE Signal Process. Mag., vol. 31, no. 3, pp. 44-53, 2014.

[16] T.G. Kolda and B.W. Bader, "Tensor decompositions and applications," SIAM Review, vol. 51, no. 3, pp. 455-500, 2009.

[17] L. Wald, T. Ranchin, and M. Mangolini, "Fusion of satellite images of different spatial resolutions: Assessing the quality of resulting images," Photogramm. Eng. Rem. S., vol. 63, no. 6, pp. 691-699, 1997.

[18] R.H. Bartels and G.W. Stewart, "Solution of the matrix equation $\mathrm{AX}+\mathrm{XB}=\mathrm{C}$," Commun. ACM, vol. 15, no. 9, pp. 820-826, 1972.

[19] G. Golub, S. Nash, and C. Van Loan, "A Hessenberg-Schur method for the problem $\mathrm{AX}+\mathrm{XB}=\mathrm{C}$," IEEE Trans. Autom. Control, vol. 24, no. 6, pp. 909-913, 1979

[20] Y. Qi, P. Comon, and Lim L-H., "Semialgebraic geometry of nonnegative tensor rank," SIAM J. Matrix Anal. Appl., vol. 37, no. 4, pp. 1556-1580, 2016.

[21] R. Dian, L. Fang, and S. Li, "Hyperspectral image super-resolution via non-local sparse tensor factorization," IEEE Conf. on Comput. Vision and Pattern Recogn. (CVPR), p. 5344 5353, 2017.

[22] A.J. Laub, Matrix Analysis For Scientists And Engineers, Society for Industrial and Applied Mathematics, Philadelphia, PA, USA, 2004.

[23] X. Guo, S. Miron, D. Brie, and A. Stegeman, "Uni-mode and partial uniqueness conditions for CANDECOMP/PARAFAC of three-way arrays with linearly dependent loadings," SIAM J. Matrix Anal. Appl., vol. 33, no. 1, pp. 111-129, 2012.

[24] L. De Lathauwer, "Decompositions of a higher-order tensor in block termspart II: Definitions and uniqueness," SIAM J. Matrix Anal. Appl., vol. 30, no. 3, pp. 1033-1066, 2008.

[25] D. Kressner, M. Steinlechner, and B. Vandereycken, "Low-rank tensor completion by Riemannian optimization," BIT Numer. Math., vol. 54 no. 2, pp. 447-468, 2014

[26] C. I. Kanatsoulis, X. Fu, N. D. Sidiropoulos, and M. Akçakaya, "Tensor Completion from Regular Sub-Nyquist Samples," arXiv e-prints, p. arXiv:1903.00435, Mar 2019.

[27] N. Vervliet, O. Debals, L. Sorber, M. Van Barel, and L. De Lathauwer, "Tensorlab 3.0," Mar. 2016, Available online.

[28] "Grupo de inteligencia computacional (gic)," http://www.ehu.eus/ ccwintco/index.php/Hyperspectral_Remote_Sensing_Scenes accessed: 2018-10-23.

[29] E. Sanchez and B.R. Kowalski, "Tensorial resolution: a direct trilinear decomposition," J. Chemom., vol. 4, no. 1, pp. 29-45, 1990.

[30] "HSR via tensor decomposition (github repository)," https://github.com/ marhar19/HSR_via_tensor_decomposition. accessed: 2019-04-11.

[31] Q. Wei, N. Dobigeon, and J.-Y. Tourneret, "Fast fusion of multi-band images based on solving a Sylvester equation," IEEE Trans. Image Process., vol. 24, no. 11, pp. 41094121, 2015.

[32] P. Comon, "Tensors versus matrices, usefulness and unexpected properties," IEEE Workshop on Stat. Signal Process., pp. 780-788, Sep 2009.

[33] P. Comon, G. Golub, L-H. Lim, and B. Mourrain, "Symmetric tensors and symmetric tensor rank," SIAM J. Matrix Anal. Appl., Soc. Ind. Appl. Math., vol. 30, no. 3, pp. 1254-1279, 2008.

[34] J. Brachat, P. Comon, B. Mourrain, and E.P. Tsigaridas, "Symmetric tensor decomposition," 17th European Signal Process. Conf. 2009, pp. 525-529, Aug 2009.

[35] P. Comon, "Tensor decompositions, state of the art and applications," in Mathematics in Signal Processing V, J. G. McWhirter and I. K. Proudler, Eds., pp. 1-24. Clarendon Press, Oxford, UK, 2002, available at arxiv:0905.0454.

[36] V. Simoncini, "Computational methods for linear matrix equations," SIAM Review, vol. 58, no. 3, pp. 377-441, 2016. 